\title{
Schrödinger Operators with Reverse Hölder Class Potentials in the Dunkl Setting and Their Hardy Spaces
}

\section{Agnieszka Hejna ${ }^{1}$}

Received: 2 September 2020 / Revised: 15 March 2021 / Accepted: 15 March 2021 /

Published online: 4 May 2021

(C) The Author(s) 2021

\section{Abstract}

For a normalized root system $R$ in $\mathbb{R}^{N}$ and a multiplicity function $k \geq 0$ let $\mathbf{N}=$ $N+\sum_{\alpha \in R} k(\alpha)$. We denote by $d w(\mathbf{x})=\Pi_{\alpha \in R}|\langle\mathbf{x}, \alpha\rangle|^{k(\alpha)} d \mathbf{x}$ the associated measure in $\mathbb{R}^{N}$. Let $L=-\Delta+V, V \geq 0$, be the Dunkl-Schrödinger operator on $\mathbb{R}^{N}$. Assume that there exists $q>\max \left(1, \frac{\mathbf{N}}{2}\right)$ such that $V$ belongs to the reverse Hölder class $\mathrm{RH}^{q}(d w)$. We prove the Fefferman-Phong inequality for $L$. As an application, we conclude that the Hardy space $H_{L}^{1}$, which is originally defined by means of the maximal function associated with the semigroup $e^{-t L}$, admits an atomic decomposition with local atoms in the sense of Goldberg, where their localizations are adapted to $V$.

Keywords Rational Dunkl theory · Schrödinger operators · Reverse Hölder classes · Fefferman-Phong inequality $\cdot$ Hardy spaces

Mathematics Subject Classification 42B30 $\cdot$ 42B25 $\cdot 42 \mathrm{~B} 35 \cdot 35 \mathrm{~K} 08 \cdot 35 \mathrm{~J} 10$

\section{Introduction}

On $\mathbb{R}^{N}, N \geq 3$, let us consider Schrödinger differential operator

$$
\mathscr{L}=-\Delta_{\text {eucl }}+V(x)=-\sum_{j=1}^{N} \partial_{j}^{2}+V(x)
$$

Communicated by Pencho Petrushev.

Research supported by the National Science Centre, Poland (Narodowe Centrum Nauki), Grant 2017/25/B/ST1/00599.

$凶 \quad$ Agnieszka Hejna

hejna@math.uni.wroc.pl

1 Instytut Matematyczny, Uniwersytet Wrocławski, Pl. Grunwaldzki 2/4, 50-384 Wrocław, Poland 
where $V \in L_{\text {loc }}^{2}\left(\mathbb{R}^{N}, d x\right)$ is a non-negative potential which belongs to the reverse Hölder class $B_{q}$ with $q>\frac{N}{2}$, i.e. the inequality

$$
\left(\frac{1}{|B|} \int_{B} V(x)^{q} d x\right)^{1 / q} \leq C \frac{1}{|B|} \int_{B} V(x) d x
$$

holds for every ball $B$ in $\mathbb{R}^{N}$. Define the auxiliary function $\mathbf{m}$ as follows:

$$
\frac{1}{\mathbf{m}(x)}=\sup \left\{r>0: \frac{1}{r^{N-2}} \int_{B(x, r)} V(x) d x \leq 1\right\} .
$$

The integral defining the function $\mathbf{m}$ was introduced by Ch. Fefferman (see [20, p. 146, the assumption of the main lemma]). The function is then used in the well-known Fefferman-Phong inequality ( [20, p. 146], see also Shen [35], [36, Lemma 1.9]) which we state below.

Theorem 1 (Fefferman-Phong inequality) There is a constant $C>0$ such that for all $f \in C_{c}^{1}\left(\mathbb{R}^{N}\right)$ we have

$$
\int_{\mathbb{R}^{N}} \mathbf{m}(x)^{2}|f(x)|^{2} d x \leq C\left(\sum_{j=1}^{N} \int_{\mathbb{R}^{N}}\left|\partial_{j} f(x)\right|^{2} d x+\int_{\mathbb{R}^{N}} V(x)|f(x)|^{2} d x\right) .
$$

The proof of (4) is based on the usage of the fact that $V \in A_{p}$ for some $p>1$ and on the Poincaré inequality

$$
\frac{1}{|B(x, r)|} \int_{B(x, R)}\left|f(y)-f_{B(x, r)}\right|^{2} d y \leq \frac{C r^{2}}{|B(x, r)|} \int_{B(x, r)}|\nabla f(y)|^{2} d x .
$$

The Fefferman-Phong inequality and the function $\mathbf{m}$ itself are very useful tools which are used in analysis regarding the operator $\mathscr{L}$, e.g., in investigating behavior of its eigenvalues [20], estimating of the fundamental solution of $\mathscr{L}$ ( [36, Theorem 2.7]) and studying $L^{p}$-bounds of the operators $\nabla \mathscr{L}^{i \gamma}, \nabla \mathscr{L}^{-1 / 2}, \nabla \mathscr{L}^{-1} \nabla, \nabla^{2} \mathscr{L}^{-1}$ (see Theorems $0.3,0.4,0.5,0.8$ in [36]). It was proved in [15] (see also [16, Theorem 2.11, Proposition 2.16]) that the integral kernel $k_{t}(x, y)$ of the Schrödinger semigroup $e^{-t \mathscr{L}}$ behaves like the classical heat semigroup for $0<t<\mathbf{m}(x)^{-2}$, while for $t>\mathbf{m}(x)^{-2}$ has essentially faster decay. These observations allowed Dziubański and Zienkiewicz [15] to study the Hardy spaces associated with $\mathscr{L}$ and prove a local character of atoms (see also [17,18]).

The aim of this article is to prove the Fefferman-Phong inequality for DunklSchrödinger operators and study its applications for describing behavior of the corresponding Dunkl-Schrödinger semigroups and their Hardy spaces $H^{1}$.

The Dunkl theory is a generalization of the Euclidean Fourier analysis. It started with the seminal article [10] and developed extensively afterwards (see e.g. [8,9,11,12,21, 29-31,38,39]). We refer the reader to lecture notes [32] and [33] for more information and references. We fix a normalized root system $R$ in $\mathbb{R}^{N}$ and a multiplicity function 
$k \geq 0$ (see Sect. 2). For $\xi \in \mathbb{R}^{N}, N \geq 1$, the Dunkl operators $T_{\xi}$ are the following $k$-deformations of the directional derivatives $\partial_{\xi}$ by a difference operator:

$$
T_{\xi} f(\mathbf{x})=\partial_{\xi} f(\mathbf{x})+\sum_{\alpha \in R} \frac{k(\alpha)}{2}\langle\alpha, \xi\rangle \frac{f(\mathbf{x})-f\left(\sigma_{\alpha}(\mathbf{x})\right)}{\langle\alpha, \mathbf{x}\rangle},
$$

where $\sigma_{\alpha}$ is the reflection on $\mathbb{R}^{N}$ with respect to the hyperspace orthogonal to $\alpha$. The Dunkl operators are generalizations of the directional derivatives (in fact, they are ordinary partial derivatives for $k \equiv 0$ ), however they are non-local operators. Therefore, in order to obtain counterparts of classical Euclidean harmonic analysis results in the Dunkl setting, we have to deal with both: local and non-local parts of the operators under consideration. For instance, the question what would be a good counterpart of Poincare's inequality (5) in the rational Dunkl setting seems to be an interesting problem. Recently various different versions of (5) were proved (see $[27,40,41])$. The analysis is more complicated if we compose such operators. Furthermore, there are other technical problems and open questions in Dunkl theory. One of them is the lack of knowledge about boundendess of the so called Dunkl translations $\tau_{\mathbf{x}}$ on $L^{p}(d w)$ spaces for $p \neq 2$. It makes analysis of convolution operators more complicated and delicate.

In the present paper we consider the Dunkl-Schrödinger operator

$$
L=-\Delta+V \text { on } \mathbb{R}^{N}, N \geq 1
$$

where $V \in L_{\text {loc }}^{2}(d w)$ is a non-negative potential and $\Delta=\sum_{j=1}^{N} T_{e_{j}}^{2}$ is the Dunkl Laplacian. Here and subsequently, $\left\{e_{j}\right\}_{1 \leq j \leq N}$ denote the canonical orthonormal basis in $\mathbb{R}^{N}$. Such operators were recently studied by Amri and Hammi in [2] and [3]. An example of such operator is the so called Dunkl harmonic oscillator $-\Delta+\|x\|^{2}$, whose properties are better understood (see [1,24,28,29], and [33]). Let $\mathbf{N}$ be the homogeneous dimension (see (7)). We shall assume that $V$ satisfies an analogue of (2) with $q>\max \left(1, \frac{\mathbf{N}}{2}\right)$ (see Sect. 2.3 for details). In the current paper we prove that a counterpart of the Fefferman-Phong inequality (4) is true in the Dunkl setting, which is one of our main results (see Theorem 6). The main difficulty which one faces trying to prove Theorem 6 is the lack of knowledge about the Poincare's inequality, which is the main ingredient of the proof in the classical case. Our idea of the proof is to mix the methods which are known from the theory of non-local operators (see [18, proof of Theorem 9.4]), a version of pseudo-Poincare's inequality (62) (which is very close to that in [40, Section 5]), together with properties of the counterpart of the function $\mathbf{m}$, which in the Dunkl setting will be denoted here by $m$. The careful analysis of the properties of the function $m$ and the proof of Theorem 6 are the goals of Part 1 of the paper.

Part 2 is devoted to the application of the Fefferman-Phong inequality to prove the characterization of the Hardy space $H_{L}^{1}$ associated with the Dunkl-Schrödinger operator by the maximal function associated with the semigroup generated by $-\Delta+V$ and by a special atomic decomposition - see Sect. 6 (Theorem 7) for the details. This application is inspired by [15] (see also [14] and [17]). The atoms for $H_{L}^{1}$ have the 
structure of local atoms in the sense of Goldberg [23] with localization adapted to the behavior of the function $m$. So, in order to obtain our result, we need characterizations of a family local Hardy spaces in the Dunkl setting proved in [24, Section 5].

\section{Preliminaries}

\subsection{Basic Definitions of the Dunkl Theory}

In this section we present basic facts concerning the theory of the Dunkl operators. For details we refer the reader to [10,32], and [33].

We consider the Euclidean space $\mathbb{R}^{N}$ with the scalar product $\langle\mathbf{x}, \mathbf{y}\rangle=\sum_{j=1}^{N} x_{j} y_{j}$, where $\mathbf{x}=\left(x_{1}, \ldots, x_{N}\right), \mathbf{y}=\left(y_{1}, \ldots, y_{N}\right)$, and the norm $\|\mathbf{x}\|^{2}=\langle\mathbf{x}, \mathbf{x}\rangle$. For a nonzero vector $\alpha \in \mathbb{R}^{N}$, the reflection $\sigma_{\alpha}$ with respect to the hyperplane $\alpha^{\perp}$ orthogonal to $\alpha$ is given by

$$
\sigma_{\alpha}(\mathbf{x})=\mathbf{x}-2 \frac{\langle\mathbf{x}, \alpha\rangle}{\|\alpha\|^{2}} \alpha
$$

In this paper we fix a normalized root system in $\mathbb{R}^{N}$, that is, a finite set $R \subset \mathbb{R}^{N} \backslash\{0\}$ such that $R \cap \alpha \mathbb{R}=\{ \pm \alpha\}, \sigma_{\alpha}(R)=R$, and $\|\alpha\|=\sqrt{2}$ for all $\alpha \in R$. The finite group $G$ generated by the reflections $\sigma_{\alpha} \in R$ is called the Weyl group (reflection group) of the root system. A multiplicity function is a $G$-invariant function $k: R \rightarrow \mathbb{C}$ which will be fixed and $\geq 0$ throughout this paper. Let

$$
d w(\mathbf{x})=\prod_{\alpha \in R}|\langle\mathbf{x}, \alpha\rangle|^{k(\alpha)} d \mathbf{x}
$$

be the associated measure in $\mathbb{R}^{N}$, where, here and subsequently, $d \mathbf{x}$ stands for the Lebesgue measure in $\mathbb{R}^{N}$. We denote by

$$
\mathbf{N}=N+\sum_{\alpha \in R} k(\alpha)
$$

the homogeneous dimension of the system. Clearly,

$$
w(B(t \mathbf{x}, t r))=t^{\mathbf{N}} w(B(\mathbf{x}, r)) \text { for all } \mathbf{x} \in \mathbb{R}^{N}, t, r>0,
$$

where $B(\mathbf{x}, r)=\left\{\mathbf{y} \in \mathbb{R}^{N}:\|\mathbf{y}-\mathbf{x}\|<r\right\}$. Moreover,

$$
\int_{\mathbb{R}^{N}} f(\mathbf{x}) d w(\mathbf{x})=\int_{\mathbb{R}^{N}} t^{-\mathbf{N}} f(\mathbf{x} / t) d w(\mathbf{x}) \text { for } f \in L^{1}(d w) \text { and } t>0 .
$$

Observe that there is a constant $C>0$ such that

$$
C^{-1} w(B(\mathbf{x}, r)) \leq r^{N} \prod_{\alpha \in R}(|\langle\mathbf{x}, \alpha\rangle|+r)^{k(\alpha)} \leq C w(B(\mathbf{x}, r)),
$$


so $d w(\mathbf{x})$ is doubling, that is, there is a constant $C>0$ such that

$$
w(B(\mathbf{x}, 2 r)) \leq C w(B(\mathbf{x}, r)) \text { for all } \mathbf{x} \in \mathbb{R}^{N}, r>0 .
$$

Moreover, there exists a constant $C \geq 1$ such that, for every $\mathbf{x} \in \mathbb{R}^{N}$ and for all $r_{2} \geq r_{1}>0$

$$
C^{-1}\left(\frac{r_{2}}{r_{1}}\right)^{N} \leq \frac{w\left(B\left(\mathbf{x}, r_{2}\right)\right)}{w\left(B\left(\mathbf{x}, r_{1}\right)\right)} \leq C\left(\frac{r_{2}}{r_{1}}\right)^{\mathbf{N}}
$$

For a measurable subset $A$ of $\mathbb{R}^{N}$ we define

$$
\mathcal{O}(A)=\left\{\sigma_{\alpha}(\mathbf{x}): \mathbf{x} \in A, \alpha \in R\right\}
$$

Clearly, by (8), for all $\mathbf{x} \in \mathbb{R}^{N}$ and $r>0$ we get

$$
w(\mathcal{O}(B(\mathbf{x}, r))) \leq|G| w(B(\mathbf{x}, r))
$$

For $\xi \in \mathbb{R}^{N}$, the Dunkl operators $T_{\xi}$ are the following $k$-deformations of the directional derivatives $\partial_{\xi}$ by a difference operator:

$$
T_{\xi} f(\mathbf{x})=\partial_{\xi} f(\mathbf{x})+\sum_{\alpha \in R} \frac{k(\alpha)}{2}\langle\alpha, \xi\rangle \frac{f(\mathbf{x})-f\left(\sigma_{\alpha}(\mathbf{x})\right)}{\langle\alpha, \mathbf{x}\rangle} .
$$

The Dunkl operators $T_{\xi}$, which were introduced in [10], commute and are skewsymmetric with respect to the $G$-invariant measure $d w$.

For fixed $\mathbf{y} \in \mathbb{R}^{N}$ the Dunkl kernel $E(\mathbf{x}, \mathbf{y})$ is the unique analytic solution to the system

$$
T_{\xi} f=\langle\xi, \mathbf{y}\rangle f, \quad f(0)=1 .
$$

The function $E(\mathbf{x}, \mathbf{y})$, which generalizes the exponential function $e^{\langle\mathbf{x}, \mathbf{y}\rangle}$, has the unique extension to a holomorphic function on $\mathbb{C}^{N} \times \mathbb{C}^{N}$. Moreover, it satisfies $E(\mathbf{x}, \mathbf{y})=$ $E(\mathbf{y}, \mathbf{x})$ for all $\mathbf{x}, \mathbf{y} \in \mathbb{C}^{N}$.

Let $\left\{e_{j}\right\}_{1 \leq j \leq N}$ denotes the canonical orthonormal basis in $\mathbb{R}^{N}$ and let $T_{j}=T_{e_{j}}$. In our further consideration we shall need the following lemma.

Lemma 1 For all $\mathbf{x} \in \mathbb{R}^{N}, \mathbf{z} \in \mathbb{C}^{N}$ and $v \in \mathbb{N}_{0}^{N}$ we have

$$
\left|\partial_{\mathbf{z}}^{\nu} E(\mathbf{x}, \mathbf{z})\right| \leq\|\mathbf{x}\|^{|v|} \exp (\|\mathbf{x}\|\|\operatorname{Re} \mathbf{z}\|) .
$$

In particular,

$$
|E(i \xi, \mathbf{x})| \leq 1 \quad \text { for all } \xi, \mathbf{x} \in \mathbb{R}^{N}
$$

Proof See [30, Corollary 5.3]. 
Corollary 1 There is a constant $C>0$ such that for all $\mathbf{x}, \xi \in \mathbb{R}^{N}$ we have

$$
|E(i \xi, \mathbf{x})-1| \leq C\|\mathbf{x}\|\|\xi\|
$$

The Dunkl transform

$$
\mathcal{F} f(\xi)=c_{k}^{-1} \int_{\mathbb{R}^{N}} E(-i \xi, \mathbf{x}) f(\mathbf{x}) d w(\mathbf{x}),
$$

where

$$
c_{k}=\int_{\mathbb{R}^{N}} e^{-\frac{\|\mathbf{x}\|^{2}}{2}} d w(\mathbf{x})>0
$$

originally defined for $f \in L^{1}(d w)$, is an isometry on $L^{2}(d w)$, i.e.,

$$
\|f\|_{L^{2}(d w)}=\|\mathcal{F} f\|_{L^{2}(d w)} \text { for all } f \in L^{2}(d w),
$$

and preserves the Schwartz class of functions $\mathcal{S}\left(\mathbb{R}^{N}\right)$ (see [7]). Its inverse $\mathcal{F}^{-1}$ has the form

$$
\mathcal{F}^{-1} g(x)=c_{k}^{-1} \int_{\mathbb{R}^{N}} E(i \xi, \mathbf{x}) g(\xi) d w(\xi)
$$

Moreover,

$$
\mathcal{F}\left(T_{j} f\right)(\xi)=i \xi_{j} \mathcal{F} f(\xi)
$$

The Dunkl translation $\tau_{\mathbf{x}} f$ of a function $f \in \mathcal{S}\left(\mathbb{R}^{N}\right)$ by $\mathbf{x} \in \mathbb{R}^{N}$ is defined by

$$
\tau_{\mathbf{x}} f(\mathbf{y})=c_{k}^{-1} \int_{\mathbb{R}^{N}} E(i \xi, \mathbf{x}) E(i \xi, \mathbf{y}) \mathcal{F} f(\xi) d w(\xi) .
$$

It is a contraction on $L^{2}(d w)$, however it is an open problem if the Dunkl translations are bounded operators on $L^{p}(d w)$ for $p \neq 2$.

The Dunkl convolution $f * g$ of two reasonable functions (for instance Schwartz functions) is defined by

$(f * g)(\mathbf{x})=c_{k} \mathcal{F}^{-1}[(\mathcal{F} f)(\mathcal{F} g)](\mathbf{x})=\int_{\mathbb{R}^{N}}(\mathcal{F} f)(\xi)(\mathcal{F} g)(\xi) E(\mathbf{x}, i \xi) d w(\xi)$ for $\mathbf{x} \in \mathbb{R}^{N}$

or, equivalently, by

$$
(f * g)(\mathbf{x})=\int_{\mathbb{R}^{N}} f(\mathbf{y}) \tau_{\mathbf{x}} g(-\mathbf{y}) d w(\mathbf{y})=\int_{\mathbb{R}^{N}} f(\mathbf{y}) g(\mathbf{x}, \mathbf{y}) d w(\mathbf{y}) \text { for all } \mathbf{x} \in \mathbb{R}^{N},
$$

where, here and subsequently, $g(\mathbf{x}, \mathbf{y})=\tau_{\mathbf{x}} g(-\mathbf{y})$. 


\subsection{Dunkl Laplacian and Dunkl Heat Semigroup}

The Dunkl Laplacian associated with $R$ and $k$ is the differential-difference operator $\Delta=\sum_{j=1}^{N} T_{j}^{2}$, which acts on $C^{2}\left(\mathbb{R}^{N}\right)$-functions by

$$
\begin{gathered}
\Delta f(\mathbf{x})=\Delta_{\text {eucl }} f(\mathbf{x})+\sum_{\alpha \in R} k(\alpha) \delta_{\alpha} f(\mathbf{x}), \\
\delta_{\alpha} f(\mathbf{x})=\frac{\partial_{\alpha} f(\mathbf{x})}{\langle\alpha, \mathbf{x}\rangle}-\frac{\|\alpha\|^{2}}{2} \frac{f(\mathbf{x})-f\left(\sigma_{\alpha} \mathbf{x}\right)}{\langle\alpha, \mathbf{x}\rangle^{2}} .
\end{gathered}
$$

Obviously, $\mathcal{F}(\Delta f)(\xi)=-\|\xi\|^{2} \mathcal{F} f(\xi)$. The operator $\Delta$ is essentially self-adjoint on $L^{2}(d w)$ (see for instance [2, Theorem 3.1]) and generates the semigroup $H_{t}$ of linear self-adjoint contractions on $L^{2}(d w)$. The semigroup has the form

$$
H_{t} f(\mathbf{x})=\mathcal{F}^{-1}\left(e^{-t\|\xi\|^{2}} \mathcal{F} f(\xi)\right)(\mathbf{x})=\int_{\mathbb{R}^{N}} h_{t}(\mathbf{x}, \mathbf{y}) f(\mathbf{y}) d w(\mathbf{y}),
$$

where the heat kernel

$$
h_{t}(\mathbf{x}, \mathbf{y})=\tau_{\mathbf{x}} h_{t}(-\mathbf{y}), \quad h_{t}(\mathbf{x})=\mathcal{F}^{-1}\left(e^{-t\|\xi\|^{2}}\right)(\mathbf{x})=c_{k}^{-1}(2 t)^{-\mathbf{N} / 2} e^{-\|\mathbf{x}\|^{2} /(4 t)}
$$

is a $C^{\infty}$-function of all variables $\mathbf{x}, \mathbf{y} \in \mathbb{R}^{N}, t>0$, and satisfies

$$
0<h_{t}(\mathbf{x}, \mathbf{y})=h_{t}(\mathbf{y}, \mathbf{x}), \quad \int_{\mathbb{R}^{N}} h_{t}(\mathbf{x}, \mathbf{y}) d w(\mathbf{y})=1 .
$$

Let

$$
d(\mathbf{x}, \mathbf{y})=\min _{\sigma \in G}\|\sigma(\mathbf{x})-\mathbf{y}\|
$$

be the distance of the orbit of $\mathbf{x}$ to the orbit of $\mathbf{y}$. Let us denote

$$
\mathcal{G}_{t}(\mathbf{x}, \mathbf{y})=(\max (w(B(\mathbf{x}, \sqrt{t})), w(B(\mathbf{y}, \sqrt{t}))))^{-1} \exp \left(-\frac{d(\mathbf{x}, \mathbf{y})^{2}}{t}\right) .
$$

We shall need the following estimates for $h_{t}(\mathbf{x}, \mathbf{y})$ - their two step proof, which is based on Rösler's formula for the Dunkl translations of radial functions (see [31]), can be found in [5, Theorem 4.1] and [13, Theorem 3.1].

Theorem 2 There are constants $C, c>0$ such that for all $\mathbf{x}, \mathbf{y} \in \mathbb{R}^{N}$ and $t>0$ we have

$$
h_{t}(\mathbf{x}, \mathbf{y}) \leq C\left(1+\frac{\|\mathbf{x}-\mathbf{y}\|}{\sqrt{t}}\right)^{-2} \mathcal{G}_{t / c}(\mathbf{x}, \mathbf{y})
$$

Theorem 2 implies the following Lemma (see [13, Corollary 3.5]). 
Lemma 2 Suppose that $\varphi \in C_{c}^{\infty}\left(\mathbb{R}^{N}\right)$ is radial and supported by the unit ball $B(0,1)$. Set $\varphi_{t}(\mathbf{x})=t^{-\mathbf{N}} \varphi\left(t^{-1} \mathbf{x}\right)$. Then there is $C>0$ such that for all $\mathbf{x}, \mathbf{y} \in \mathbb{R}^{N}$ and $t>0$ we have

$$
\left|\tau_{\mathbf{x}} \varphi_{t}(-\mathbf{y})\right| \leq C\left(1+\frac{\|\mathbf{x}-\mathbf{y}\|}{t}\right)^{-2}(\max (w(B(\mathbf{x}, t)), w(B(\mathbf{y}, t))))^{-1} \chi_{[0,1]}(d(\mathbf{x}, \mathbf{y}) / t) .
$$

\subsection{Dunkl-Schrödinger Operator and Semigroup}

Let $V \geq 0$ be a measurable function such that $V \in L_{\text {loc }}^{2}(d w)$. We consider the following operator on the Hilbert space $L^{2}(d w)$ :

$$
\mathcal{L}=-\Delta+V
$$

with the domain

$$
\mathcal{D}(\mathcal{L})=\left\{f \in L^{2}(d w):\|\xi\|^{2} \mathcal{F} f(\xi) \in L^{2}(d w(\xi)) \text { and } V(\mathbf{x}) f(\mathbf{x}) \in L^{2}(d w(\mathbf{x}))\right\}
$$

(see [2]). We call this operator the Dunkl-Schrödinger operator. Let us define the quadratic form

$$
\mathbf{Q}(f, g)=\sum_{j=1}^{N} \int_{\mathbb{R}^{N}} T_{j} f(\mathbf{x}) \overline{T_{j} g(\mathbf{x})} d w(\mathbf{x})+\int_{\mathbb{R}^{N}} V(\mathbf{x}) f(\mathbf{x}) \overline{g(\mathbf{x})} d w(\mathbf{x})
$$

with the domain

$$
\mathcal{D}(\mathbf{Q})=\left\{f \in L^{2}(d w):\left(\sum_{j=1}^{N}\left|T_{j} f\right|^{2}\right)^{1 / 2}, V^{1 / 2} f \in L^{2}(d w)\right\} .
$$

The quadratic form is densely defined and closed (see [2, Lemma 4.1]), so there exists a unique positive self-adjoint operator $L$ such that

$$
\langle L f, f\rangle=\mathbf{Q}(f, f) \text { for all } f \in \mathcal{D}(L),
$$

moreover,

$$
\mathcal{D}\left(L^{1 / 2}\right)=\mathcal{D}(\mathbf{Q}) \text { and } \mathbf{Q}(f, f)=\left\|L^{1 / 2} f\right\|_{L^{2}(d w)},
$$

where $L^{1 / 2}$ is a unique self-adjoint operator such that $\left(L^{1 / 2}\right)^{2}=L$. It was proved in [2, Theorem 4.6], that $\mathcal{L}$ is essentially self-adjoint on $C_{c}^{\infty}\left(\mathbb{R}^{N}\right)$ and $L$ is its closure. Consequently, $L$ generates the semigroup of self-adjoint contractions on $L^{2}(d w)$. The semigroup has the form (see [2, Theorem 4.8])

$$
K_{t} f(\mathbf{x})=\int_{\mathbb{R}^{N}} k_{t}(\mathbf{x}, \mathbf{y}) f(\mathbf{y}) d w(\mathbf{y}),
$$


where $k_{t}(\mathbf{x}, \mathbf{y})$ is the integral kernel which satisfies

$$
0 \leq k_{t}(\mathbf{x}, \mathbf{y}) \leq h_{t}(\mathbf{x}, \mathbf{y})
$$

\section{Part 1: Fefferman-Phong inequality in the rational Dunkl setting}

\section{Reverse Hölder Class}

In this part, we assume that $q>\max \left(1, \frac{\mathbf{N}}{2}\right)$ and $V \geq 0$ belongs to the reverse Hölder class $\mathrm{RH}^{q}(d w)$, that is, there is a constant $C_{\mathrm{RH}}>0$ such that

$$
\left(\frac{1}{w(B)} \int_{B} V(\mathbf{x})^{q} d w(\mathbf{x})\right)^{1 / q} \leq C_{\mathrm{RH}} \frac{1}{w(B)} \int_{B} V(\mathbf{x}) d w(\mathbf{x}) \text { for every ball } B
$$

For any Lebesque measurable set $A$ we define

$$
\mu(A)=\int_{A} V(\mathbf{x}) d w(\mathbf{x})
$$

Our goal is to study the properties of the measure $\mu$. The proofs of the results in this section are standard and they are based on the [22, Chapter 7]. For the convenience of the reader we present details.

Lemma 3 For all balls $B \subset \mathbb{R}^{N}$ and measurable sets $E \subseteq B$ we have

$$
\frac{\mu(E)}{\mu(B)} \leq C_{R H}\left(\frac{w(E)}{w(B)}\right)^{1 / q^{\prime}}
$$

where, here and subsequently, $\frac{1}{q}+\frac{1}{q^{\prime}}=1$.

Proof Applying Hölder's inequality, then the reverse Hölder inequality (27), we get

$$
\begin{aligned}
& \mu(E)=\int_{\mathbb{R}^{N}} \chi_{E}(\mathbf{x}) V(\mathbf{x}) d w(\mathbf{x}) \leq w(E)^{1 / q^{\prime}}\left(\int_{B} V(\mathbf{x})^{q} d w(\mathbf{x})\right)^{1 / q} \\
& \leq C_{\mathrm{RH}}\left(\frac{w(E)}{w(B)}\right)^{1 / q^{\prime}} \mu(B) .
\end{aligned}
$$

Lemma 4 Let $\varepsilon>0$. There is a constant $0<\gamma<1$ such that for all $\mathbf{x} \in \mathbb{R}^{N}$ and $r>0$ we have

$$
1-\frac{w(B(\mathbf{x}, \gamma r))}{w(B(\mathbf{x}, r))}=\frac{w(B(\mathbf{x}, r) \backslash B(\mathbf{x}, \gamma r))}{w(B(\mathbf{x}, r))}<\varepsilon
$$


Proof Thanks to (6) we obtain

$$
\begin{aligned}
w(B(\mathbf{x}, r) \backslash B(\mathbf{x}, \gamma r)) & =\int_{B(\mathbf{x}, r) \backslash B(\mathbf{x}, \gamma r)} \prod_{\alpha \in R}|\langle\mathbf{y}, \alpha\rangle|^{k(\alpha)} d \mathbf{y} \\
& \leq \int_{B(\mathbf{x}, r) \backslash B(\mathbf{x}, \gamma r)} \prod_{\alpha \in R}(|\langle\mathbf{y}-\mathbf{x}, \alpha\rangle|+|\langle\mathbf{x}, \alpha\rangle|)^{k(\alpha)} d \mathbf{y} .
\end{aligned}
$$

For all $\mathbf{y} \in B(\mathbf{x}, r)$ we have $|\langle\mathbf{y}-\mathbf{x}, \alpha\rangle| \leq \sqrt{2} r$, so

$$
\begin{aligned}
w(B(\mathbf{x}, r) \backslash B(\mathbf{x}, \gamma r)) & \leq 2^{\mathbf{N} / 2} \int_{B(\mathbf{x}, r) \backslash B(\mathbf{x}, \gamma r)} \prod_{\alpha \in R}(|\langle\mathbf{x}, \alpha\rangle|+r)^{k(\alpha)} d \mathbf{y} \\
& =v_{N} 2^{\mathbf{N} / 2}\left(r^{N}-\gamma^{N} r^{N}\right) \prod_{\alpha \in R}(|\langle\mathbf{x}, \alpha\rangle|+r)^{k(\alpha)},
\end{aligned}
$$

where $v_{N}$ is the Euclidean measure of the unit $N$-dimensional ball. Consequently, thanks to (8), we have

$$
\frac{w(B(\mathbf{x}, r) \backslash B(\mathbf{x}, \gamma r))}{w(B(\mathbf{x}, r))} \leq C\left(1-\gamma^{N}\right),
$$

where the constant $C>0$ is independent of $\mathbf{x}$ and $r$. The claim follows easily.

Lemma 5 The measure $\mu$ defined in (28) is doubling, i.e. there is a constant $C_{\mu}>0$ such that for all $\mathbf{x} \in \mathbb{R}$ and $r>0$ we have

$$
\mu(B(\mathbf{x}, 2 r)) \leq C_{\mu} \mu(B(\mathbf{x}, r)) .
$$

Proof Let $0<\gamma<1$. Setting $B=B(\mathbf{x}, r)$ and $E=B(\mathbf{x}, r) \backslash B(\mathbf{x}, \gamma r)$ in (29), we get

$$
1-\frac{\mu(B(\mathbf{x}, \gamma r))}{\mu(B(\mathbf{x}, r))} \leq C_{\mathrm{RH}}\left(1-\frac{w(B(\mathbf{x}, \gamma r))}{w(B(\mathbf{x}, r))}\right)^{1 / q^{\prime}}
$$

Thanks to Lemma 4 for $1-\gamma$ small enough we have

$$
C_{\mathrm{RH}}\left(1-\frac{w(B(\mathbf{x}, \gamma r))}{w(B(\mathbf{x}, r))}\right)^{1 / q^{\prime}}<1 / 2
$$

consequently,

$$
\mu(B(\mathbf{x}, r)) \leq 2 \mu(B(\mathbf{x}, \gamma r))
$$

There is $n \in \mathbb{N}$ such that $\gamma^{n}<1 / 2$. Applying (31) $n$ times we get the claim. 
As the consequence of the doubling property of $\mu$, we obtain the following corollary.

Corollary 2 There is a constant $C_{R H}^{\prime}>0$ such that for all cubes $Q \subset \mathbb{R}^{N}$ and measurable sets $E \subseteq Q$ we have

$$
\begin{aligned}
& \left(\frac{1}{w(Q)} \int_{Q} V(\mathbf{x})^{q} d w(\mathbf{x})\right)^{1 / q} \leq C_{R H}^{\prime} \frac{1}{w(Q)} \int_{Q} V(\mathbf{x}) d w(\mathbf{x}), \\
& \frac{\mu(E)}{\mu(Q)} \leq C_{R H}^{\prime}\left(\frac{w(E)}{w(Q)}\right)^{1 / q^{\prime}} .
\end{aligned}
$$

Lemma 6 There are $0<\gamma, \delta<1$ such that for all cubes $Q \subset \mathbb{R}^{N}$ and measurable sets $E \subseteq Q$ the following implication is true:

$$
\mu(E)<\gamma \mu(Q) \Rightarrow w(E)<\delta w(Q) .
$$

Proof Set $\gamma^{\prime}>0$ small enough in order to have $\delta^{\prime}=C_{\mathrm{RH}}^{\prime}\left(\gamma^{\prime}\right)^{1 / q^{\prime}}<1$, where $C_{\mathrm{RH}}^{\prime}$ is the constant in (33). Then, by (33), we have the implication

$$
w(E) \leq \gamma^{\prime} w(Q) \Rightarrow \mu(E) \leq \delta^{\prime} \mu(Q) .
$$

Taking $Q \backslash E$ instead of $E$ in (35) we get

$$
w(E) \geq\left(1-\gamma^{\prime}\right) w(Q) \Rightarrow \mu(E) \geq\left(1-\delta^{\prime}\right) \mu(Q) .
$$

Note that (36) is equivalent to (34) with $\gamma=1-\delta^{\prime}$ and $\delta=1-\gamma^{\prime}$.

We will need the following classical result from theory of the $A_{p}$ weights (see [22, Corollary 7.2.4]).

Proposition 1 Let $v$ be the weight and let $v$ be a doubling measure on $\mathbb{R}^{N}$. Suppose that there are $0<\gamma, \delta<1$ such that

$$
\nu(E)<\gamma \nu(Q) \Rightarrow \int_{E} v(\mathbf{x}) d \nu(\mathbf{x})<\delta \int_{Q} v(\mathbf{x}) d v(\mathbf{x}),
$$

whenever $E$ is a $\nu$-measurable subset of a cube $Q$. Then there are constants $C, \eta>0$ such that for every cube $Q$ in $\mathbb{R}^{N}$ we have

$$
\left(\frac{1}{v(Q)} \int_{Q} v^{1+\eta}(\mathbf{x}) d v(\mathbf{x})\right)^{1 /(1+\eta)} \leq C \frac{1}{v(Q)} \int_{Q} v(\mathbf{x}) d v(\mathbf{x}) .
$$

Proposition 2 There is a constant $C>0$ and the exponent $p>1$ such that for every cube $Q$ in $\mathbb{R}^{N}$ we have

$$
\left(\frac{1}{w(Q)} \int_{Q} V(\mathbf{x}) d w(\mathbf{x})\right)\left(\frac{1}{w(Q)} \int_{Q} V^{-\frac{1}{p-1}}(\mathbf{x}) d w(\mathbf{x})\right)^{p-1} \leq C .
$$


Proof Note that (34) is equivalent to

$$
\mu(E)<\gamma \mu(Q) \Rightarrow \int_{E} V^{-1}(\mathbf{x}) d \mu(\mathbf{x})<\delta \int_{Q} V^{-1}(\mathbf{x}) d \mu(\mathbf{x}) .
$$

Hence, applying Proposition 1 to $v=V^{-1}$ and $v=\mu$ (the assumption that $v$ is doubling is satisfied thanks to Lemma 5) we get that there are $C, \eta>0$ such that

$$
\left(\frac{1}{\mu(Q)} \int_{Q} V(\mathbf{x})^{-1-\eta} V(\mathbf{x}) d w(\mathbf{x})\right)^{1 /(1+\eta)} \leq C \frac{1}{\mu(Q)} \int_{Q} V(\mathbf{x})^{-1} V(\mathbf{x}) d w(\mathbf{x})=C \frac{w(Q)}{\mu(Q)}
$$

Finally, it can be checked that (40) is equivalent to (38) with $p=1+\frac{1}{\eta}$.

Here and subsequently, we write

$$
\gamma=2-\frac{\mathbf{N}}{q}
$$

The reverse Hölder inequality (27) has the following consequence (see [36, Lemma $1.2]$ ), which will be used in the next section many times.

Lemma 7 Assume that $V \in \mathrm{RH}^{q}(d w)$, where $q>\max \left(1, \frac{\mathbf{N}}{2}\right)$, and $V \geq 0$. There is a constant $C \geq 1$ such that for all $\mathbf{x} \in \mathbb{R}^{N}$ and $0<r_{1}<r_{2}<\infty$ we have

$$
\frac{r_{1}^{2}}{w\left(B\left(\mathbf{x}, r_{1}\right)\right)} \int_{B\left(\mathbf{x}, r_{1}\right)} V(\mathbf{y}) d w(\mathbf{y}) \leq C\left(\frac{r_{1}}{r_{2}}\right)^{\gamma} \frac{r_{2}^{2}}{w\left(B\left(\mathbf{x}, r_{2}\right)\right)} \int_{B\left(\mathbf{x}, r_{2}\right)} V(\mathbf{y}) d w(\mathbf{y})
$$

Proof Thanks to Hölder's inequality and the reverse Hölder inequality (27), we get

$$
\begin{aligned}
& \frac{1}{w\left(B\left(\mathbf{x}, r_{1}\right)\right)} \int_{B\left(\mathbf{x}, r_{1}\right)} V(\mathbf{y}) d w(\mathbf{y}) \leq\left(\frac{1}{w\left(B\left(\mathbf{x}, r_{1}\right)\right)} \int_{B\left(\mathbf{x}, r_{1}\right)} V(\mathbf{y})^{q} d w(\mathbf{y})\right)^{1 / q} \\
& \leq \frac{w\left(B\left(\mathbf{x}, r_{2}\right)\right)^{1 / q}}{w\left(B\left(\mathbf{x}, r_{1}\right)\right)^{1 / q}}\left(\frac{1}{w\left(B\left(\mathbf{x}, r_{2}\right)\right)} \int_{B\left(\mathbf{x}, r_{2}\right)} V(\mathbf{y})^{q} d w(\mathbf{y})\right)^{1 / q} \\
& \leq C_{\mathrm{RH}} \frac{w\left(B\left(\mathbf{x}, r_{2}\right)\right)^{1 / q}}{w\left(B\left(\mathbf{x}, r_{1}\right)\right)^{1 / q}} \frac{1}{w\left(B\left(\mathbf{x}, r_{2}\right)\right)} \int_{B\left(\mathbf{x}, r_{2}\right)} V(\mathbf{y}) d w(\mathbf{y}) .
\end{aligned}
$$

Finally, the claim follows from (10). 


\section{The Auxiliary Function $m(x)$}

\subsection{Definition and Growth Properties of $m(x)$}

For $\mathbf{x} \in \mathbb{R}^{N}$ we define

$$
\frac{1}{m(\mathbf{x})}=\sup \left\{r>0: \frac{r^{2}}{w(B(\mathbf{x}, r))} \int_{B(\mathbf{x}, r)} V(\mathbf{y}) d w(\mathbf{y}) \leq 1\right\}
$$

(see [36, Definition 1.3]). Thanks to Lemma 7, for all $\mathbf{x} \in \mathbb{R}^{N}$ (and $V \not \equiv 0$ ) we have $\lim _{r \rightarrow 0} \frac{r^{2}}{w(B(\mathbf{x}, r))} \int_{B(\mathbf{x}, r)} V(\mathbf{y}) d w(\mathbf{y})=0, \quad \lim _{r \rightarrow+\infty} \frac{r^{2}}{w(B(\mathbf{x}, r))} \int_{B(\mathbf{x}, r)} V(\mathbf{y}) d w(\mathbf{y})=+\infty$,

so the function $m$ is well-defined and $0<m(\mathbf{x})<\infty$. The next lemma is an adaptation of [36, Lemma 1.4].

Lemma 8 Assume that $V \in \mathrm{RH}^{q}(d w)$, where $q>\max \left(1, \frac{\mathbf{N}}{2}\right)$, and $V \geq 0$. There are constants $C, \kappa>0$ such that for all $\mathbf{x}, \mathbf{y} \in \mathbb{R}^{N}$ we have

$$
\begin{aligned}
& C^{-1} m(\mathbf{y}) \leq m(\mathbf{x}) \leq C m(\mathbf{y}) \text { if }\|\mathbf{x}-\mathbf{y}\|<m(\mathbf{x})^{-1}, \\
& m(\mathbf{y}) \leq C m(\mathbf{x})(1+m(\mathbf{x})\|\mathbf{x}-\mathbf{y}\|)^{\kappa}, \\
& m(\mathbf{y}) \geq C^{-1} m(\mathbf{x})(1+m(\mathbf{x})\|\mathbf{x}-\mathbf{y}\|)^{-\frac{\kappa}{1+\kappa}}
\end{aligned}
$$

Proof of (44) By the doubling property of $w$ and $\mu$ we have $w(B(\mathbf{x}, r)) \sim w(B(\mathbf{y}, r))$ and $\mu(B(\mathbf{x}, r)) \sim \mu(B(\mathbf{y}, r))$ if $r \geq\|\mathbf{x}-\mathbf{y}\|$. So, by Lemma 7 , for any $r<m(\mathbf{x})^{-1}$ we have

$$
\begin{aligned}
& \frac{r^{2}}{w(B(\mathbf{y}, r))} \int_{B(\mathbf{y}, r)} V(\mathbf{z}) d w(\mathbf{z}) \\
& \leq C\left(\frac{r}{m(\mathbf{x})^{-1}}\right)^{\gamma} \frac{m(\mathbf{x})^{-2}}{w\left(B\left(\mathbf{y}, m(\mathbf{x})^{-1}\right)\right)} \int_{B\left(\mathbf{y}, m(\mathbf{x})^{-1}\right)} V(\mathbf{z}) d w(\mathbf{z}) \\
& \leq C^{\prime}\left(\frac{r}{m(\mathbf{x})^{-1}}\right)^{\gamma} \frac{m(\mathbf{x})^{-2}}{w\left(B\left(\mathbf{x}, m(\mathbf{x})^{-1}\right)\right)} \int_{B\left(\mathbf{x}, m(\mathbf{x})^{-1}\right)} V(\mathbf{z}) d w(\mathbf{z}) \\
& \leq C^{\prime}\left(\frac{r}{m(\mathbf{x})^{-1}}\right)^{\gamma},
\end{aligned}
$$

where in the last inequality we have used the definition of $m$. Note that (47) implies that for

$$
r<\min \left(1,\left(2 C^{\prime}\right)^{-\gamma^{-1}}\right) m(\mathbf{x})^{-1},
$$


we get

$$
\frac{r^{2}}{w(B(\mathbf{y}, r))} \int_{B(\mathbf{y}, r)} V(\mathbf{z}) d w(\mathbf{z}) \leq \frac{1}{2}
$$

so the inequality $m(\mathbf{y}) \leq C m(\mathbf{x})$ follows. Now we turn to the proof of $m(\mathbf{x}) \leq C m(\mathbf{y})$. For $r>2 m(\mathbf{x})^{-1}$, thanks to the doubling property of $\mu$ and $w$, then by Lemma 7, we write

$$
\begin{aligned}
& \frac{r^{2}}{w(B(\mathbf{y}, r))} \int_{B(\mathbf{y}, r)} V(\mathbf{z}) d w(\mathbf{z}) \geq C \frac{r^{2}}{w(B(\mathbf{x}, r))} \int_{B(\mathbf{x}, r)} V(\mathbf{z}) d w(\mathbf{z}) \\
& \quad \geq C^{\prime}\left(\frac{r}{2 m(\mathbf{x})^{-1}}\right)^{\gamma} \frac{\left(2 m(\mathbf{x})^{-1}\right)^{2}}{w\left(B\left(\mathbf{x}, 2 m(\mathbf{x})^{-1}\right)\right)} \int_{B\left(\mathbf{x}, 2 m(\mathbf{x})^{-1}\right)} V(\mathbf{z}) d w(\mathbf{z}) \geq C^{\prime}\left(\frac{r}{m(\mathbf{x})^{-1}}\right)^{\gamma}
\end{aligned}
$$

where in the last inequality we have used the definition of $m(\mathbf{x})$. Taking

$$
r>\max \left(2,\left(C^{\prime} / 2\right)^{-\gamma^{-1}}\right) m(\mathbf{x})^{-1}
$$

we have

$$
\frac{r^{2}}{w(B(\mathbf{y}, r))} \int_{B(\mathbf{y}, r)} V(\mathbf{z}) d w(\mathbf{z}) \geq 2,
$$

so, thanks to the definition of $m$ (see (42)), the proof is complete.

Proof of (45) We may assume $\|\mathbf{x}-\mathbf{y}\| m(\mathbf{x}) \geq 1$, otherwise the claim follows from (44). Let $r=m(\mathbf{x})^{-1}$ and let $j \geq 1, j \in \mathbb{Z}$, be such that

$$
2^{j-1} r<\|\mathbf{x}-\mathbf{y}\| \leq 2^{j} r
$$

Let $0<r_{1}<r$. Thanks to Lemma 7 , then the doubling property of $\mu$ and $w$ together with (10), we have

$$
\begin{aligned}
& \frac{r_{1}^{2}}{w\left(B\left(\mathbf{y}, r_{1}\right)\right)} \int_{B\left(\mathbf{y}, r_{1}\right)} V(\mathbf{z}) d w(\mathbf{z}) \\
& \leq C\left(\frac{r_{1}}{\|\mathbf{x}-\mathbf{y}\|}\right)^{\gamma} \frac{\|\mathbf{x}-\mathbf{y}\|^{2}}{w(B(\mathbf{y},\|\mathbf{x}-\mathbf{y}\|))} \int_{B(\mathbf{y},\|\mathbf{x}-\mathbf{y}\|)} V(\mathbf{z}) d w(\mathbf{z}) \\
& \quad \leq C\left(\frac{r_{1}}{\|\mathbf{x}-\mathbf{y}\|}\right)^{\gamma} \frac{\|\mathbf{x}-\mathbf{y}\|^{2}}{w(B(\mathbf{x},\|\mathbf{x}-\mathbf{y}\|))} \int_{B(\mathbf{x},\|\mathbf{x}-\mathbf{y}\|)} V(\mathbf{z}) d w(\mathbf{z}) \\
& \quad \leq C\left(\frac{r_{1}}{2^{j} r}\right)^{\gamma} 2^{-j N} C_{\mu}^{j} \frac{2^{2 j} r^{2}}{w(B(\mathbf{x}, r))} \int_{B(\mathbf{x}, r)} V(\mathbf{z}) d w(\mathbf{z}) \leq C\left(\frac{r_{1}}{2^{j} r}\right)^{\gamma} 2^{j(2-N)} C_{\mu}^{j},
\end{aligned}
$$


where $C_{\mu}$ is the doubling constant for $\mu$ (see Lemma 5). Therefore, there is a constant $C_{1}>1$ independent of $\mathbf{x}, \mathbf{y} \in \mathbb{R}^{N}$ and $r>r_{1}>0$ such that if $r_{1} \leq r C_{1}^{-j}$, then

$$
\frac{r_{1}^{2}}{w\left(B\left(\mathbf{y}, r_{1}\right)\right)} \int_{B\left(\mathbf{y}, r_{1}\right)} V(\mathbf{z}) d w(\mathbf{z}) \leq C\left(\frac{r_{1}}{2^{j} r}\right)^{\gamma} 2^{-j N} C_{\mu}^{j} 2^{2 j} \leq \frac{1}{2} .
$$

Consequently, by the definition of $m(\mathbf{y})$ we have

$$
\frac{1}{m(\mathbf{y})} \geq r C_{1}^{-j}=\frac{1}{m(\mathbf{x})} C_{1}^{-j}
$$

which leads us to

$$
m(\mathbf{y}) \leq m(\mathbf{x}) C_{1}^{j} \leq C m(\mathbf{x})(1+m(\mathbf{x})\|\mathbf{x}-\mathbf{y}\|)^{\kappa}
$$

where $\kappa=\log _{2} C_{1}$.

Proof of (46) We may assume that $\|\mathbf{x}-\mathbf{y}\| \geq m(\mathbf{y})^{-1}$, otherwise the claim follows from (44). By (45) we have

$$
m(\mathbf{x}) \leq C m(\mathbf{y})(1+\|\mathbf{x}-\mathbf{y}\| m(\mathbf{y}))^{\kappa} \leq C m(\mathbf{y})^{1+\kappa}\|\mathbf{x}-\mathbf{y}\|^{\kappa} .
$$

Thus,

$$
m(\mathbf{y}) \geq C^{\prime} \frac{m(\mathbf{x})^{1 /(1+\kappa)}}{\|\mathbf{x}-\mathbf{y}\|^{\kappa /(1+\kappa)}} \geq C \frac{m(\mathbf{x})}{(1+m(\mathbf{x})\|\mathbf{x}-\mathbf{y}\|)^{\kappa /(1+\kappa)}},
$$

so the proof is complete.

\subsection{Associated Collection of Cubes $\mathcal{Q}$}

For a cube $Q \subset \mathbb{R}^{N}$, here and subsequently, let $d(Q)$ denote the side-length of cube $Q$. We denote by $Q^{*}$ the cube with the same center as $Q$ such that $d\left(Q^{*}\right)=2 d(Q)$. We define a collection of dyadic cubes $\mathcal{Q}$ associated with the potential $V$ by the following stopping-time condition:

$Q \in \mathcal{Q} \Longleftrightarrow Q$ is the maximal dyadic cube for which $\frac{d(Q)^{2}}{w(Q)} \int_{Q} V(\mathbf{y}) d w(\mathbf{y}) \leq 1$

Fact 3 The collection $\mathcal{Q}$ is well-defined and it forms a covering of $\mathbb{R}^{N}$ built from dyadic cubes which have disjoint interiors. 
Proof Note that for any $\mathbf{x} \notin \bigcup_{j \in \mathbb{Z}} 2^{j} \mathbb{Z}^{N}$ there is a unique sequence $\left\{Q_{j}\right\}_{j \in \mathbb{Z}}$ of dyadic cubes such that $\mathbf{x} \in Q_{j}$ satisfying

$$
Q_{j} \subset Q_{j+1} \text { and } d\left(Q_{j}\right)=2^{j} \text { for all } j \in \mathbb{Z} \text {. }
$$

Further, if follows from (43) and the doubling property of measures $d \mu$ and $d w$ that

$$
\lim _{j \rightarrow-\infty} \frac{d\left(Q_{j}\right)^{2}}{w\left(Q_{j}\right)} \int_{Q_{j}} V(\mathbf{y}) d w(\mathbf{y})=0 \text { and } \lim _{j \rightarrow \infty} \frac{d\left(Q_{j}\right)^{2}}{w\left(Q_{j}\right)} \int_{Q_{j}} V(\mathbf{y}) d w(\mathbf{y})=\infty
$$

Thus the collection given by (48) is well-defined and it forms the desired covering of $\mathbb{R}^{N}$.

We list below simple facts about the collection $\mathcal{Q}$, which are consequences of properties of $w, \mu$ and $m(\mathbf{x})$.

Fact 4 Assume that $V \in \mathrm{RH}^{q}(d w)$, where $q>\max \left(1, \frac{\mathbf{N}}{2}\right)$, and $V \geq 0$. There is a constant $C>0$ such that for any $Q \in \mathcal{Q}$ we have

$$
C^{-1} \leq \frac{d(Q)^{2}}{w(Q)} \int_{Q} V(\mathbf{x}) d w(\mathbf{x}) \leq 1 .
$$

Proof It is an easy consequence of the doubling property of $\mu$. Namely, let $\widetilde{Q}$ be the parent of cube $Q \in \mathcal{Q}$. As the consequence of the stopping-time condition (48), we get

$1<\frac{d(\widetilde{Q})^{2}}{w(\widetilde{Q})} \int_{\widetilde{Q}} V(\mathbf{x}) d w(\mathbf{x}) \leq \frac{(2 d(Q))^{2}}{w(Q)} \int_{\widetilde{Q}} V(\mathbf{x}) d w(\mathbf{x}) \leq C \frac{d(Q)^{2}}{w(Q)} \int_{Q} V(\mathbf{x}) d w(\mathbf{x})$.

Fact 5 Assume that $V \in \mathrm{RH}^{q}(d w)$, where $q>\max \left(1, \frac{\mathbf{N}}{2}\right)$, and $V \geq 0$. There is $a$ constant $C>0$ such that for any $Q \in \mathcal{Q}$ and $\mathbf{x} \in Q^{* * * *}$ we have

$$
C^{-1} d(Q)^{-1} \leq m(\mathbf{x}) \leq C d(Q)^{-1} .
$$

Proof The proof is essentially the same as that of (44). We provide the details. The doubling property of $\mu$ and $w$ combined with (49) imply that there is $C^{\prime} \geq 1$ such that

$$
\frac{1}{C^{\prime}} \leq \frac{d(Q)^{2}}{w(B(\mathbf{x}, d(Q))} \int_{B(\mathbf{x}, d(Q))} V(\mathbf{y}) d w(\mathbf{y}) \leq C^{\prime} \text { for all } \mathbf{x} \in Q^{* * * *}
$$

Using Lemma 7 with $r_{1}=d(Q)$ and any $r_{2} \geq\left(C^{\prime} C\right)^{1 / \gamma} d(Q)$, we get

$$
1 \leq \frac{r_{2}^{2}}{w\left(B\left(\mathbf{x}, r_{2}\right)\right)} \int_{B\left(\mathbf{x}, r_{2}\right)} V(\mathbf{y}) d w(\mathbf{y})
$$


which implies $m(\mathbf{x})^{-1} \leq\left(C C^{\prime}\right)^{1 / \gamma} d(Q)$. Similarly, applying again Lemma 7 , this time with $r_{1}=\left(C C^{\prime}\right)^{-1 / \gamma} d(Q)$ and $r_{2}=d(Q)$, we obtain

$$
\frac{r_{1}^{2}}{w\left(B\left(\mathbf{x}, r_{1}\right)\right)} \int_{B\left(\mathbf{x}, r_{1}\right)} V(\mathbf{y}) d w(\mathbf{y}) \leq 1,
$$

which leads to $m(\mathbf{x})^{-1} \geq r_{1}=\left(C C^{\prime}\right)^{\gamma} d(Q)$.

Lemma 8 together with Fact 5 imply the following proposition.

Proposition 3 Assume that $V \in \mathrm{RH}^{q}(d w)$, where $q>\max \left(1, \frac{\mathbf{N}}{2}\right)$, and $V \geq 0$. The covering $\mathcal{Q}$ defined by (48) satisfies the following finite overlapping condition:

$\left(\exists C_{0}>0\right)\left(\forall Q_{1}, Q_{2} \in \mathcal{Q}\right) Q_{1}^{* * * *} \cap Q_{2}^{* * * *} \neq \emptyset \Rightarrow C_{0}^{-1} d\left(Q_{1}\right) \leq d\left(Q_{2}\right) \leq C_{0} d\left(Q_{1}\right)$.

\section{Fefferman-Phong Inequality in the Dunkl Setting}

The goal of this section is the prove the Fefferman-Phong inequality in the rational Dunkl setting. This result is crucial in the proof of condition (D) (see Sect. 6) for potential satisfying (27). The result for $k \equiv 0$ is due to C. Feffermann and D.H. Phong [20] (see also [36, Lemma 1.9]). The proof is inspired by one from [18, Theorem 9.4].

Theorem 6 (Fefferman-Phong type inequality) Assume that $V \in \mathrm{RH}^{q}(d w)$, where $q>\max \left(1, \frac{\mathbf{N}}{2}\right)$, and $V \geq 0$. There is a constant $C>0$ such that for all $f \in \mathcal{D}(\mathbf{Q})$ we have

$$
\int_{\mathbb{R}^{N}}|f(\mathbf{x})|^{2} m(\mathbf{x})^{2} d w(\mathbf{x}) \leq C \mathbf{Q}(f, f) .
$$

We need some lemmas before providing the proof of Theorem 6.

Lemma 9 Assume that $V \in \mathrm{RH}^{q}(d w)$, where $q>\max \left(1, \frac{\mathbf{N}}{2}\right)$, and $V \geq 0$. There are constants $C, \eta>0$ such that for all $Q \in \mathcal{Q}$ and $\varepsilon>0$ we have $w\left(E_{\varepsilon}\right) \leq C \varepsilon^{\eta} w\left(Q^{*}\right)$, where

$$
E_{\varepsilon}=\left\{\mathbf{y} \in Q^{*}: V(\mathbf{y}) \leq \varepsilon d(Q)^{-2}\right\}
$$

Proof Let $p>1$ be the number from (38). By the definition of $E_{\varepsilon}$ we write

$$
\begin{aligned}
w\left(E_{\varepsilon}\right)^{p-1} & =\left(\int_{E_{\varepsilon}} d w(\mathbf{y})\right)^{p-1} \leq\left(\int_{E_{\varepsilon}} \varepsilon^{1 /(p-1)} d(Q)^{-2 /(p-1)} V(\mathbf{y})^{-\frac{1}{p-1}} d w(\mathbf{y})\right)^{p-1} \\
& \leq \varepsilon d(Q)^{-2}\left(\int_{Q^{*}} V(\mathbf{y})^{-\frac{1}{p-1}} d w(\mathbf{y})\right)^{p-1}
\end{aligned}
$$


Thanks to (49) and the doubling property of $w$ we have

$$
d(Q)^{-2} \leq C \frac{1}{w(Q)} \int_{Q} V(\mathbf{y}) d w(\mathbf{y}) \leq C^{\prime} \frac{1}{w\left(Q^{*}\right)} \int_{Q^{*}} V(\mathbf{y}) d w(\mathbf{y}) .
$$

Consequently, applying (55) and (56) together with (38) we get

$$
\begin{aligned}
w\left(E_{\varepsilon}\right)^{p-1} & \leq C^{\prime} \varepsilon\left(\frac{1}{w\left(Q^{*}\right)} \int_{Q^{*}} V(\mathbf{y}) d w(\mathbf{y})\right)\left(\int_{Q^{*}} V(\mathbf{y})^{-\frac{1}{p-1}} d w(\mathbf{y})\right)^{p-1} \\
& \leq C \varepsilon w\left(Q^{*}\right)^{p-1} .
\end{aligned}
$$

Lemma 10 For all $j \in\{1,2, \ldots, N\}, g \in C_{c}^{\infty}\left(\mathbb{R}^{N}\right)$, and $f \in L^{2}(d w)$ such that its weak Dunkl derivative $T_{j} f$ is in $L^{2}(d w)$ we have $T_{j}(f g) \in L^{2}(d w)$. Moreover,

$$
T_{j}(f g)(\mathbf{x})=\left(T_{j} f\right)(\mathbf{x}) g(\mathbf{x})+f(\mathbf{x}) \partial_{j} g(\mathbf{x})+\sum_{\alpha \in R} \frac{k(\alpha)}{2} \alpha_{j} f\left(\sigma_{\alpha}(\mathbf{x})\right) \frac{g(\mathbf{x})-g\left(\sigma_{\alpha}(\mathbf{x})\right)}{\langle\mathbf{x}, \alpha\rangle}
$$

in $L^{2}(d w)$-sense.

Proof It is a standard fact, but for the convenience of reader we provide the proof. Let us assume first that $f \in C^{1}\left(\mathbb{R}^{N}\right)$. By the definition of $T_{j}$ (see (13)) we have

$$
\begin{aligned}
& T_{j}(f g)(\mathbf{x})=\partial_{j}(f g)(\mathbf{x})+\sum_{\alpha \in R} \frac{k(\alpha)}{2} \alpha_{j} \frac{f(\mathbf{x}) g(\mathbf{x})-f\left(\sigma_{\alpha}(\mathbf{x})\right) g\left(\sigma_{\alpha}(\mathbf{x})\right)}{\langle\mathbf{x}, \alpha\rangle} \\
& =f(\mathbf{x})\left(\partial_{j} g\right)(\mathbf{x})+\left(\partial_{j} f\right)(\mathbf{x}) g(\mathbf{x})+\sum_{\alpha \in R} \frac{k(\alpha)}{2} \alpha_{j} g(\mathbf{x}) \frac{f(\mathbf{x})-f\left(\sigma_{\alpha}(\mathbf{x})\right)}{\langle\mathbf{x}, \alpha\rangle} \\
& \quad+\sum_{\alpha \in R} \frac{k(\alpha)}{2} \alpha_{j} f\left(\sigma_{\alpha}(\mathbf{x})\right) \frac{g(\mathbf{x})-g\left(\sigma_{\alpha}(\mathbf{x})\right)}{\langle\mathbf{x}, \alpha\rangle} \\
& =f(\mathbf{x}) \partial_{j} g(\mathbf{x})+\left(T_{j} f\right)(\mathbf{x}) g(\mathbf{x})+\sum_{\alpha \in R} \frac{k(\alpha)}{2} \alpha_{j} f\left(\sigma_{\alpha}(\mathbf{x})\right) \frac{g(\mathbf{x})-g\left(\sigma_{\alpha}(\mathbf{x})\right)}{\langle\mathbf{x}, \alpha\rangle} .
\end{aligned}
$$

In order to obtain the general case, let us take $\psi \in C_{c}^{\infty}\left(\mathbb{R}^{N}\right)$. By the definition of $T_{j}(f g)$ and (58) we have

$$
\begin{gathered}
\int_{\mathbb{R}^{N}} T_{j}(f g)(\mathbf{x}) \psi(\mathbf{x}) d w(\mathbf{x})=-\int_{\mathbb{R}^{N}} f(\mathbf{x}) g(\mathbf{x}) T_{j} \psi(\mathbf{x}) d w(\mathbf{x}) \\
=-\int_{\mathbb{R}^{N}} f(\mathbf{x}) T_{j}(g \psi)(\mathbf{x})+\int_{\mathbb{R}^{N}} f(\mathbf{x}) \partial_{j} g(\mathbf{x}) \psi(\mathbf{x}) d w(\mathbf{x}) \\
\quad+\sum_{\alpha \in R} \frac{k(\alpha)}{2} \alpha_{j} \int_{\mathbb{R}^{N}} f(\mathbf{x}) \psi\left(\sigma_{\alpha}(\mathbf{x})\right) \frac{g(\mathbf{x})-g\left(\sigma_{\alpha}(\mathbf{x})\right)}{\langle\mathbf{x}, \alpha\rangle} d w(\mathbf{x})
\end{gathered}
$$




$$
\begin{aligned}
= & \int_{\mathbb{R}^{N}} T_{j} f(\mathbf{x}) g(\mathbf{x}) \psi(\mathbf{x}) d w(\mathbf{x})+\int_{\mathbb{R}^{N}} f(\mathbf{x}) \partial_{j} g(\mathbf{x}) \psi(\mathbf{x}) d w(\mathbf{x}) \\
& +\sum_{\alpha \in R} \frac{k(\alpha)}{2} \alpha_{j} \int_{\mathbb{R}^{N}} f\left(\sigma_{\alpha}(\mathbf{x})\right) \frac{g(\mathbf{x})-g\left(\sigma_{\alpha}(\mathbf{x})\right)}{\langle\mathbf{x}, \alpha\rangle} \psi(\mathbf{x}) d w(\mathbf{x}) .
\end{aligned}
$$

Definition 1 Assume that $\widetilde{\mathcal{Q}}$ is a covering of $\mathbb{R}^{N}$ by the cubes with pairwise disjoint interiors and it satisfies the finite overlapping condition (52). By the smooth resolution of identity $\left\{\phi_{Q}\right\}_{Q \in \mathcal{\mathcal { Q }}}$ associated with $\widetilde{\mathcal{Q}}$ we mean the collection of $C^{\infty}$-functions on $\mathbb{R}^{N}$ such that $\operatorname{supp} \phi_{Q} \subseteq Q^{*}, 0 \leq \phi_{Q}(\mathbf{x}) \leq 1$,

$$
\left|\partial^{\alpha} \phi_{Q}(\mathbf{x})\right| \leq C_{\alpha} d(Q)^{-|\alpha|} \text { for all } \alpha \in \mathbb{N}_{0}^{N}
$$

and $\sum_{Q \in \widetilde{\mathcal{Q}}} \phi_{Q}(\mathbf{x})=1$ for all $\mathbf{x} \in \mathbb{R}^{N}$.

In this part of the paper, we consider the smooth resolution of identity associated with $\mathcal{Q}$. The existence of $\left\{\phi_{Q}\right\}_{Q \in \mathcal{Q}}$ is guaranteed by (52) (see Proposition 3).

Lemma 11 There is a constant $C>0$ such that for all $\alpha \in R, Q \in \mathcal{Q}$, and $\mathbf{x} \in Q^{*}$ we have

$$
\left|\frac{\phi_{Q}(\mathbf{x})-\phi_{Q}\left(\sigma_{\alpha}(\mathbf{x})\right)}{\langle\mathbf{x}, \alpha\rangle}\right| \leq C d(Q)^{-1} .
$$

Proof This is the standard fact - we write

$$
\begin{aligned}
\frac{\phi_{Q}(\mathbf{x})-\phi_{Q}\left(\sigma_{\alpha}(\mathbf{x})\right)}{\langle\mathbf{x}, \alpha\rangle} & =-\frac{1}{\langle\mathbf{x}, \alpha\rangle} \int_{0}^{1} \frac{d}{d t} \phi_{Q}\left(\mathbf{x}-2 t \frac{\langle\mathbf{x}, \alpha\rangle}{\|\alpha\|^{2}} \alpha\right) d t \\
& =\int_{0}^{1}\left\langle\left(\nabla_{\mathbf{x}} \phi_{Q}\right)\left(\mathbf{x}-2 t \frac{\langle\mathbf{x}, \alpha\rangle}{\|\alpha\|^{2}} \alpha\right), \alpha\right\rangle d t
\end{aligned}
$$

so the claim is a consequence of (59).

Lemma 12 Assume that $V \in \mathrm{RH}^{q}(d w)$, where $q>\max \left(1, \frac{\mathbf{N}}{2}\right)$, and $V \geq 0$. There is a constant $C>0$ such that for all $j \in\{1, \ldots, N\}, f \in L^{2}(d w)$ such that its weak Dunkl derivative $T_{j} f$ is in $L^{2}(d w)$, and $Q \in \mathcal{Q}$ we have

$$
\begin{aligned}
\left\|T_{j}\left(f \phi_{Q}\right)\right\|_{L^{2}(d w)} \leq & C\left(\left(\int_{Q^{*}}\left|T_{j} f(\mathbf{x})\right|^{2} d w(\mathbf{x})\right)^{1 / 2}\right. \\
& \left.+\left(\int_{\mathcal{O}\left(Q^{*}\right)}|f(\mathbf{x})|^{2} m(\mathbf{x})^{2} d w(\mathbf{x})\right)^{1 / 2}\right) .
\end{aligned}
$$

Let us remind that $\mathcal{O}\left(Q^{*}\right)$ denotes the orbit of the cube $Q^{*}$, see (11). 
Proof By Lemma 10 we have

$$
\begin{aligned}
\left\|T_{j}\left(f \phi_{Q}\right)\right\|_{L^{2}(d w)} & \leq\left\|\left(T_{j} f\right)(\mathbf{x}) \phi_{Q}(\mathbf{x})\right\|_{L^{2}(d w(\mathbf{x}))}+\left\|f(\mathbf{x})\left(\partial_{j} \phi_{Q}\right)(\mathbf{x})\right\|_{L^{2}(d w(\mathbf{x}))} \\
& +C \sum_{\alpha \in R}\left\|f\left(\sigma_{\alpha}(\mathbf{x})\right) \frac{\phi_{Q}(\mathbf{x})-\phi_{Q}\left(\sigma_{\alpha}(\mathbf{x})\right)}{\langle\mathbf{x}, \alpha\rangle}\right\|_{L^{2}(d w(\mathbf{x}))}
\end{aligned}
$$

Thanks to the property that $\operatorname{supp} \phi_{Q} \subseteq Q^{*},(59)$, and Fact 5 we have

$$
\begin{aligned}
& \left\|\left(T_{j} f\right)(\mathbf{x}) \phi_{Q}(\mathbf{x})\right\|_{L^{2}(d w(\mathbf{x}))} \leq C\left(\int_{Q^{*}}\left|T_{j} f(\mathbf{x})\right|^{2} d w(\mathbf{x})\right)^{1 / 2}, \\
& \left\|f(\mathbf{x})\left(\partial_{j} \phi_{Q}\right)(\mathbf{x})\right\|_{L^{2}(d w(\mathbf{x}))} \leq C\left(\int_{Q^{*}}|f(\mathbf{x})|^{2} m(\mathbf{x})^{2} d w(\mathbf{x})\right)^{1 / 2} .
\end{aligned}
$$

Therefore, it is enough to estimate

$$
\begin{aligned}
& \int_{\mathcal{O}\left(Q^{*}\right)}\left|f\left(\sigma_{\alpha}(\mathbf{x})\right) \frac{\phi_{Q}(\mathbf{x})-\phi_{Q}\left(\sigma_{\alpha}(\mathbf{x})\right)}{\langle\mathbf{x}, \alpha\rangle}\right|^{2} d w(\mathbf{x}) \\
& =\int_{\mathcal{O}\left(Q^{*}\right) \cap\left\{\mathbf{x}: \sqrt{2}|\langle\mathbf{x}, \alpha\rangle| \leq m(\mathbf{x})^{-1}\right\}} \cdots \\
& \quad+\int_{\mathcal{O}\left(Q^{*}\right) \cap\left\{\mathbf{x}: \sqrt{2}|\langle\mathbf{x}, \alpha\rangle|>m(\mathbf{x})^{-1}\right\}} \cdots=: I_{1}+I_{2}
\end{aligned}
$$

for fixed $\alpha \in R$. We consider $I_{1}$ first. Let us denote

$$
E=\mathcal{O}\left(Q^{*}\right) \cap\left\{\mathbf{x}: \sqrt{2}|\langle\mathbf{x}, \alpha\rangle| \leq m(\mathbf{x})^{-1}\right\} \cap\left\{\mathbf{x}: \frac{\phi_{Q}(\mathbf{x})-\phi_{Q}\left(\sigma_{\alpha}(\mathbf{x})\right)}{\langle\mathbf{x}, \alpha\rangle} \neq 0\right\}
$$

If $\mathbf{x} \in E$, then $\mathbf{x} \in Q^{*}$ or $\sigma_{\alpha}(\mathbf{x}) \in Q^{*}$, so, by Fact $5, d(Q)^{-1} \leq C m(\mathbf{x})$ or $d(Q)^{-1} \leq$ $\mathrm{Cm}\left(\sigma_{\alpha}(\mathbf{x})\right)$ respectively. Note that

$$
\mathcal{O}\left(Q^{*}\right) \cap\left\{\mathbf{x}: \sqrt{2}|\langle\mathbf{x}, \alpha\rangle| \leq m(\mathbf{x})^{-1}\right\}=\mathcal{O}\left(Q^{*}\right) \cap\left\{\mathbf{x}:\left\|\mathbf{x}-\sigma_{\alpha}(\mathbf{x})\right\| \leq m(\mathbf{x})^{-1}\right\},
$$

so, by (44), we have

$$
d(Q)^{-1} \leq C \max \left(m(\mathbf{x}), m\left(\sigma_{\alpha}(\mathbf{x})\right)\right) \leq C^{\prime} m\left(\sigma_{\alpha}(\mathbf{x})\right) \text { for all } \mathbf{x} \in E .
$$

Consequently, by Lemma 11, we get

$$
\begin{aligned}
I_{1} & \leq \int_{E}\left|f\left(\sigma_{\alpha}(\mathbf{x})\right) \frac{\phi_{Q}(\mathbf{x})-\phi_{Q}\left(\sigma_{\alpha}(\mathbf{x})\right)}{\langle\mathbf{x}, \alpha\rangle}\right|^{2} d w(\mathbf{x}) \leq C \int_{E}\left|f\left(\sigma_{\alpha}(\mathbf{x})\right)\right|^{2} d(Q)^{-2} d w(\mathbf{x}) \\
& \leq C^{\prime} \int_{\mathcal{O}\left(Q^{*}\right)}\left|f\left(\sigma_{\alpha}(\mathbf{x})\right)\right|^{2} m\left(\sigma_{\alpha}(\mathbf{x})\right)^{2} d w(\mathbf{x})=C^{\prime} \int_{\mathcal{O}\left(Q^{*}\right)}|f(\mathbf{x})|^{2} m(\mathbf{x})^{2} d w(\mathbf{x}) .
\end{aligned}
$$


In order to estimate $I_{2}$, thanks to property $0 \leq\left|\phi_{Q}(\mathbf{x})-\phi_{Q}\left(\sigma_{\alpha}(\mathbf{x})\right)\right| \leq 2$, we write

$$
I_{2} \leq 4 \int_{\mathcal{O}\left(Q^{*}\right) \cap\left\{\mathbf{x}: \sqrt{2}|\langle\mathbf{x}, \alpha\rangle|>m(\mathbf{x})^{-1}\right\}}\left|f\left(\sigma_{\alpha}(\mathbf{x})\right)\right|^{2}|\langle\mathbf{x}, \alpha\rangle|^{-2} d w(\mathbf{x}) .
$$

By (46), for $\mathbf{x} \in \mathcal{O}\left(Q^{*}\right)$ such that $\sqrt{2}|\langle\mathbf{x}, \alpha\rangle|=\left\|\mathbf{x}-\sigma_{\alpha}(\mathbf{x})\right\|>m(\mathbf{x})^{-1}$ we have

$$
m(\mathbf{x}) \leq C m\left(\sigma_{\alpha}(\mathbf{x})\right)\left(1+m(\mathbf{x})\left\|\mathbf{x}-\sigma_{\alpha}(\mathbf{x})\right\|\right)^{\frac{\kappa}{1+\kappa}} \leq C^{\prime} m\left(\sigma_{\alpha}(\mathbf{x})\right) m(\mathbf{x})|\langle\mathbf{x}, \alpha\rangle| .
$$

Consequently,

$$
1 \leq C^{\prime} m\left(\sigma_{\alpha}(\mathbf{x})\right)|\langle\mathbf{x}, \alpha\rangle|
$$

which leads us to

$$
\begin{aligned}
I_{2} & \leq C \int_{\mathcal{O}\left(Q^{*}\right)}\left|f\left(\sigma_{\alpha}(\mathbf{x})\right)\right|^{2}|\langle\mathbf{x}, \alpha\rangle|^{-2} m\left(\sigma_{\alpha}(\mathbf{x})\right)^{2}|\langle\mathbf{x}, \alpha\rangle|^{2} d w(\mathbf{x}) \\
& =C \int_{\mathcal{O}\left(Q^{*}\right)}|f(\mathbf{x})|^{2} m(\mathbf{x})^{2} d w(\mathbf{x}),
\end{aligned}
$$

which ends the proof.

Proof of Theorem 6 Suppose first that

$$
\int_{\mathbb{R}^{N}}|f(\mathbf{x})|^{2} m(\mathbf{x})^{2} d w(\mathbf{x})<\infty .
$$

Let $\psi \in C_{c}^{\infty}\left(\mathbb{R}^{N}\right)$ be a radial non-negative function such that $\int_{\mathbb{R}^{N}} \psi d w=1$ and supp $\psi \subseteq B(0,1)$, and let $A>1$ be a large constant (it will be chosen later). For $Q \in \mathcal{Q}$ we define the following scaled version of $\psi$ :

$$
\psi_{Q}^{A}(\mathbf{x})=\left(A^{-1} d(Q)\right)^{-\mathbf{N}} \psi\left(A d(Q)^{-1} \mathbf{x}\right)
$$

It follows from Corollary 1 that

$$
|\mathcal{F} \psi(\xi)-1| \leq C\|\xi\|
$$

Consequently, by Plancherel's theorem (see (16)), (17), and Lemma 12,

$$
\begin{aligned}
& \int_{Q^{*}}\left|\psi_{Q}^{A} *\left(\phi_{Q} f\right)(\mathbf{x})-\left(\phi_{Q} f\right)(\mathbf{x})\right|^{2} d w(\mathbf{x}) \\
& \leq C A^{-2} d(Q)^{2} \sum_{j=1}^{N} \int_{\mathbb{R}^{N}}\left|T_{j}\left(\phi_{Q} f\right)(\mathbf{x})\right|^{2} d w(\mathbf{x}) \\
& \leq C^{\prime} A^{-2} d(Q)^{2}\left(\sum_{j=1}^{N} \int_{Q^{*}}\left|T_{j} f(\mathbf{x})\right|^{2} d w(\mathbf{x})+\int_{\mathcal{O}\left(Q^{*}\right)}|f(\mathbf{x})|^{2} m(\mathbf{x})^{2} d w(\mathbf{x})\right)
\end{aligned}
$$


The first inequality in (62) can be thought as a counterpart of the Poincaré inequality (cf. (5)). By the doubling property of $w$ we have $w(B(\mathbf{x}, d(Q))) \sim w(Q) \sim w\left(Q^{*}\right)$ for all $\mathbf{x} \in Q^{*}$. Applying Lemma 2, we obtain

$$
\begin{aligned}
& \int_{Q^{*}}\left|\psi_{Q}^{A} *\left(\phi_{Q} f\right)(\mathbf{x})\right|^{2} d w(\mathbf{x})=\int_{Q^{*}}\left|\int_{Q^{*}} \tau_{\mathbf{x}} \psi_{Q^{A}}^{A}(-\mathbf{y})\left(\phi_{Q} f\right)(\mathbf{y}) d w(\mathbf{y})\right|^{2} d w(\mathbf{x}) \\
& \quad \leq C \int_{Q^{*}} \frac{w(B(\mathbf{x}, d(Q)))^{2}}{w\left(B\left(\mathbf{x}, A^{-1} d(Q)\right)\right)^{2}} \frac{1}{w(B(\mathbf{x}, d(Q)))^{2}} d w(\mathbf{x})\left\|\phi_{Q} f\right\|_{L^{1}(d w)}^{2} \\
& \quad \leq C A^{2 \mathbf{N}} \frac{1}{w\left(Q^{*}\right)}\left\|\phi_{Q} f\right\|_{L^{1}(d w)}^{2}
\end{aligned}
$$

Let $\varepsilon>0$ (it will be chosen later) and let $E_{\varepsilon}$ be defined as in (54). We write

$$
\frac{A^{2 \mathbf{N}}}{w\left(Q^{*}\right)}\left\|\phi_{Q} f\right\|_{L^{1}(d w)}^{2}=\frac{A^{2 \mathbf{N}}}{w\left(Q^{*}\right)}\left\|\phi_{Q} f\right\|_{L^{1}\left(E_{\varepsilon}, d w\right)}^{2}+\frac{A^{2 \mathbf{N}}}{w\left(Q^{*}\right)}\left\|\phi_{Q} f\right\|_{L^{1}\left(Q^{*} \backslash E_{\varepsilon}, d w\right)}^{2}
$$

By the Cauchy-Schwarz inequality and Lemma 9 we have

$$
A^{2 \mathbf{N}} \frac{1}{w\left(Q^{*}\right)}\left\|\phi_{Q} f\right\|_{L^{1}\left(E_{\varepsilon}, d w\right)}^{2} \leq C A^{2 \mathbf{N}_{\varepsilon} \eta}\left\|\phi_{Q} f\right\|_{L^{2}(d w)}^{2} .
$$

Next, by the definition of $E_{\varepsilon}$ (see (54)) and the Cauchy-Schwarz inequality we get

$$
A^{2 \mathbf{N}} \frac{1}{w\left(Q^{*}\right)}\left\|\phi_{Q} f\right\|_{L^{1}\left(Q^{*} \backslash E_{\varepsilon}, d w\right)}^{2} \leq C A^{2 \mathbf{N}} d(Q)^{2} \varepsilon^{-1} \int_{Q^{*}} V(\mathbf{x})\left|\left(\phi_{Q} f\right)(\mathbf{x})\right|^{2} d w(\mathbf{x}) .
$$

Combining (63), (64), (65), and (66) we get

$$
\begin{aligned}
& \int_{Q^{*}}\left|\psi_{Q}^{A} *\left(\phi_{Q} f\right)(\mathbf{x})\right|^{2} d w(\mathbf{x}) \leq C A^{2 \mathbf{N}} \\
& \left(\varepsilon^{\eta}\left\|\phi_{Q} f\right\|_{L^{2}(d w)}^{2}+\frac{d(Q)^{2}}{\varepsilon} \int_{Q^{*}} V(\mathbf{x})\left|\left(\phi_{Q} f\right)(\mathbf{x})\right|^{2} d w(\mathbf{x})\right) .
\end{aligned}
$$

Consequently, by (62) and (67) we get

$$
\begin{aligned}
\left\|\phi_{Q} f\right\|_{L^{2}(d w)}^{2} \leq & C A^{-2} d(Q)^{2}\left(\sum_{j=1}^{N} \int_{Q^{*}}\left|T_{j} f(\mathbf{x})\right|^{2} d w(\mathbf{x})+\int_{\mathcal{O}\left(Q^{*}\right)}|f(\mathbf{x})|^{2} m(\mathbf{x})^{2} d w(\mathbf{x})\right) \\
& +C A^{2 \mathbf{N}}\left(\varepsilon^{\eta}\left\|\phi_{Q} f\right\|_{L^{2}(d w)}^{2}+\frac{d(Q)^{2}}{\varepsilon} \int_{Q^{*}} V(\mathbf{x})\left|\left(\phi_{Q} f\right)(\mathbf{x})\right|^{2} d w(\mathbf{x})\right),
\end{aligned}
$$


which, for $\varepsilon=\left(\frac{1}{2} C^{-1} A^{-2 N}\right)^{1 / \eta}$, leads us to

$$
\begin{aligned}
\left\|\phi_{Q} f\right\|_{L^{2}(d w)}^{2} \leq & C^{\prime} A^{-2} d(Q)^{2} \\
& \left(\sum_{j=1}^{N} \int_{Q^{*}}\left|T_{j} f(\mathbf{x})\right|^{2} d w(\mathbf{x})+\int_{\mathcal{O}\left(Q^{*}\right)}|f(\mathbf{x})|^{2} m(\mathbf{x})^{2} d w(\mathbf{x})\right) \\
& +C^{\prime} A^{2 \mathbf{N}} d(Q)^{2} \varepsilon^{-1} \int_{Q^{*}} V(\mathbf{x})\left|\left(\phi_{Q} f\right)(\mathbf{x})\right|^{2} d w(\mathbf{x}) .
\end{aligned}
$$

Dividing the inequality (68) by $d(Q)^{2}$ and then use Fact 5 , we obtain

$$
\begin{aligned}
& \int_{Q^{*}}\left|\left(\phi_{Q} f\right)(\mathbf{x})\right|^{2} m(\mathbf{x})^{2} d w(\mathbf{x}) \leq C A^{-2} \sum_{j=1}^{N} \int_{Q^{*}}\left|T_{j} f(\mathbf{x})\right|^{2} d w(\mathbf{x}) \\
& +C A^{-2} \int_{\mathcal{O}\left(Q^{*}\right)}|f(\mathbf{x})|^{2} m(\mathbf{x})^{2} d w(\mathbf{x})+C A^{2 \mathbf{N}_{\varepsilon}-1} \int_{Q^{*}} V(\mathbf{x})\left|\left(\phi_{Q} f\right)(\mathbf{x})\right|^{2} d w(\mathbf{x}) .
\end{aligned}
$$

Summing up over all $Q \in \mathcal{Q}$ we get

$$
\begin{aligned}
& \int_{\mathbb{R}^{N}}|f(\mathbf{x})|^{2} m(\mathbf{x})^{2} d w(\mathbf{x}) \\
& \leq C A^{-2}\left(\sum_{j=1}^{N} \int_{\mathbb{R}^{N}}\left|T_{j} f(\mathbf{x})\right|^{2} d w(\mathbf{x})+|G| \int_{\mathbb{R}^{N}}|f(\mathbf{x})|^{2} m(\mathbf{x})^{2} d w(\mathbf{x})\right) \\
& \quad+C A^{2 \mathbf{N}_{\varepsilon}} \int_{\mathbb{R}^{N}} V(\mathbf{x})|f(\mathbf{x})|^{2} d w(\mathbf{x}) .
\end{aligned}
$$

Taking into account (61) and taking $A$ large enough we obtain the claim for $f$ satisfying (61). For general case, we take a radial function $\eta \in C_{c}^{\infty}\left(\mathbb{R}^{N}\right)$ such that $0 \leq \eta \leq 1$, $\eta(\mathbf{x})=1$ for all $\|\mathbf{x}\| \leq 1, \eta(\mathbf{x})=0$ for all $\|\mathbf{x}\|>2$, and

$$
\left|\partial_{j} \eta(\mathbf{x})\right| \leq 2 \text { for all } \mathbf{x} \in \mathbb{R}^{N} \text { and } j \in\{1,2, \ldots, N\} .
$$

For $f \in \mathcal{D}(\mathbf{Q})$ and $n \in \mathbb{N}$ we define $f_{n}(\mathbf{x})=f(\mathbf{x}) \eta(\mathbf{x} / n)$. Note that by (57) we have $f_{n} \in \mathcal{D}(\mathbf{Q})$. Moreover, thanks to the fact that $f \in L^{2}(d w)$ and (45), the condition (61) is satisfied for $f_{n}$. Therefore, by (53) for $f_{n}$, we get

$$
\int_{\mathbb{R}^{N}}|f(\mathbf{x})|^{2} m(\mathbf{x})^{2} d w(\mathbf{x})=\lim _{n \rightarrow \infty} \int_{\mathbb{R}^{N}}\left|f_{n}(\mathbf{x})\right|^{2} m(\mathbf{x})^{2} d w(\mathbf{x}) \leq C \lim _{n \rightarrow \infty} \mathbf{Q}\left(f_{n}, f_{n}\right) .
$$

Clearly,

$$
\lim _{n \rightarrow \infty}\left\|f-f_{n}\right\|_{L^{2}(d w)}=0 .
$$


Recall that $f, T_{j} f \in L^{2}(d w)$. Applying Lemma 10, we have

$$
\begin{aligned}
& \lim _{n_{1}, n_{2} \rightarrow \infty} \int_{\mathbb{R}^{N}}\left|T_{j}\left(f_{n_{1}}-f_{n_{2}}\right)(\mathbf{x})\right|^{2} d w(\mathbf{x}) \\
& \leq 2 \lim _{n_{1}, n_{2} \rightarrow \infty} \int_{\mathbb{R}^{N}}\left|T_{j} f(\mathbf{x})\right|^{2}\left|\eta\left(\mathbf{x} / n_{1}\right)-\eta\left(\mathbf{x} / n_{2}\right)\right|^{2} d w(\mathbf{x}) \\
& \quad+4 \lim _{n_{1}, n_{2} \rightarrow \infty} \int_{\mathbb{R}^{N}}|f(x)|^{2}\left(\left|\partial_{j}\left(\eta\left(\mathbf{x} / n_{1}\right)\right)\right|^{2}+\left|\partial_{j}\left(\eta\left(\mathbf{x} / n_{2}\right)\right)\right|^{2}\right) d w(\mathbf{x}) \\
& \leq 2 \lim _{n_{1}, n_{2} \rightarrow \infty} \int_{\min \left(n_{1}, n_{2}\right) \leq\|\mathbf{x}\| \leq 2 \max \left(n_{1}, n_{2}\right)}\left|T_{j} f(\mathbf{x})\right|^{2} d w(\mathbf{x}) \\
& \quad+32 \lim _{n_{1}, n_{2} \rightarrow \infty} \int_{\mathbb{R}^{N}}|f(\mathbf{x})|^{2}\left(n_{1}^{-2}+n_{2}^{-2}\right) d w(\mathbf{x})=0 .
\end{aligned}
$$

Similarly, $V(\mathbf{x})^{1 / 2} f(\mathbf{x}) \in L^{2}(d w(\mathbf{x}))$, so

$$
\begin{aligned}
& \lim _{n_{1}, n_{2} \rightarrow \infty} \int_{\mathbb{R}^{N}} V(\mathbf{x})\left|\left(f_{n_{1}}-f_{n_{2}}\right)(\mathbf{x})\right|^{2} d w(\mathbf{x}) \\
& =\lim _{n_{1}, n_{2} \rightarrow \infty} \int_{\mathbb{R}^{N}} V(\mathbf{x})|f(\mathbf{x})|^{2}\left|\eta\left(\mathbf{x} / n_{1}\right)-\eta\left(\mathbf{x} / n_{2}\right)\right|^{2} d w(\mathbf{x}) \\
& \leq \lim _{n_{1}, n_{2} \rightarrow \infty} \int_{\min \left(n_{1}, n_{2}\right) \leq\|\mathbf{x}\| \leq 2 \max \left(n_{1}, n_{2}\right)} V(\mathbf{x})|f(\mathbf{x})|^{2} d w(\mathbf{x})=0 .
\end{aligned}
$$

Consequently, by (72) and (73) we have

$$
\lim _{n_{1}, n_{2} \rightarrow \infty} \mathbf{Q}\left(f_{n_{1}}-f_{n_{2}}, f_{n_{1}}-f_{n_{2}}\right)=0
$$

By [2, Lemma 4.1] the form $\mathbf{Q}$ is closed, so using (71) and (74), we get

$$
\lim _{n \rightarrow \infty} \mathbf{Q}\left(f_{n}, f_{n}\right)=\mathbf{Q}(f, f),
$$

which, thanks to (70), ends the proof.

Part 2: Hardy spaces associated with Dunkl-Schrödinger operator.

In this part we assume that $V \in L_{\text {loc }}^{2}(d w)$ (we do not assume is in the reverse Hölder class unless it is explicitly pointed out).

\section{Statement of the Results}

\subsection{Background to the Subject}

The classical real Hardy spaces $H^{p}$ in $\mathbb{R}^{N}$ occurred as boundary values of harmonic functions on $\mathbb{R}_{+} \times \mathbb{R}^{N}$ satisfying generalized Cauchy-Riemann equations together with certain $L^{p}$ bound conditions (see e.g. Stein-Weiss [37]). In the seminal paper of 
Fefferman and Stein [19] the spaces $H^{p}$ were characterized by means of real analysis. One of the possible characterization assets that a tempered distribution $f$ belongs to the $H^{p}\left(\mathbb{R}^{N}\right), 0<p<\infty$, if and only if the maximal function $\sup _{t>0}\left|\mathbf{h}_{t} * f(\mathbf{x})\right|$ belongs to $L^{p}\left(\mathbb{R}^{N}\right)$, where $\mathbf{h}_{t}$ is the heat kernel of the semigroup $e^{t \Delta_{\text {eucl }}}$. An important contribution to the theory is the atomic decomposition proved by Coifman [6] for $N=1$ and Latter [26] in higher dimensions, which says that every element of $H^{p}$ can be written as an (infinite) combination of special simple functions called atoms. These characterizations led to generalizations of the Hardy spaces on spaces of homogeneous type, in particular, to $H^{p}$ spaces associated with semigroups of linear operators. In [5] (see also [4,13]) a theory of Hardy spaces $H^{1}$ in the rational Dunkl setting parallel to the classical one was developed. The purpose of the remaining part of the paper is to study an $H_{L}^{1}$ space related to $L$. Our starting definition is that by means of the maximal function for the semigroup $e^{-t L}$. Then we shall prove that the space admits a special atomic decomposition. This result generalizes one of [24] where $H_{L}^{1}$ for the Dunkl harmonic oscillator $-\Delta+\|\mathbf{x}\|^{2}$ was consider. In [25] the authors provided a general approach to the theory of Hardy spaces associated with semigroups satisfying Davies-Gaffney estimates and in particular Gaussian bounds. We want to emphasize that the integral kernel for the Dunkl-Laplace semigroup does not satisfy the Gaussian bounds. Therefore the methods developed in [25] cannot be directly applied. One may ask whether the Hardy space $H_{L}^{1}$ can be characterized by the Riesz transforms $T_{j} L^{-1 / 2}$. The affirmative solution will appear in a forthcoming article.

\subsection{Hardy Spaces Associated with $L$}

Let us introduce the notion of the Hardy space associated with the operator $L$.

Definition 2 Let $f \in L^{1}(d w)$. We say that $f$ belongs to the Hardy space $H_{L}^{1}$ associated with operator $L$ if and only if

$$
f^{*}(\mathbf{x})=\sup _{t>0}\left|K_{t} f(\mathbf{x})\right|
$$

belongs to $L^{1}(d w)$. The norm in the space is given by

$$
\|f\|_{H_{L}^{1}}=\left\|f^{*}\right\|_{L^{1}(d w)}
$$

Let $\mathcal{Q}$ be a collection of closed cubes with parallel sides whose interiors are disjoint such that $\bigcup_{Q \in \mathcal{Q}} Q=\mathbb{R}^{N}$. Let us remind that $d(Q)$ denotes the side-length of cube $Q$ and we denote by $Q^{*}$ the cube with the same center as $Q$ such that $d\left(Q^{*}\right)=2 d(Q)$. Assume that this family satisfies the following finite overlapping condition:

$$
\left(\exists C_{0}>0\right)\left(\forall Q_{1}, Q_{2} \in \mathcal{Q}\right) Q_{1}^{* * * *} \cap Q_{2}^{* * * *} \neq \emptyset \Rightarrow C_{0}^{-1} d\left(Q_{1}\right) \leq d\left(Q_{2}\right) \leq C_{0} d\left(Q_{1}\right)
$$

We define the atomic Hardy space associated with the collection $\mathcal{Q}$ (see [18]). 
Definition 3 A measurable function $a(\mathbf{x})$ is called an atom for the Hardy space $H_{\mathcal{Q}}^{1 \text { at }}$ associated with the collection of cubes $\mathcal{Q}$ if

(A) supp $a \subseteq B\left(\mathbf{x}_{0}, r\right) \subseteq Q^{* * * *}$ for some $Q \in \mathcal{Q}, \mathbf{x}_{0} \in \mathbb{R}^{N}$, and $r>0$,

(B) $\sup _{\mathbf{y} \in \mathbb{R}^{N}}|a(\mathbf{y})| \leq w\left(B\left(\mathbf{x}_{0}, r\right)\right)^{-1}$,

(C) if $r<d(Q)$, then $\int_{\mathbb{R}^{N}} a(\mathbf{x}) d w(\mathbf{x})=0$.

The atomic Hardy space $H_{\mathcal{Q}}^{1 \text {, at }}$ associated with the collection $\mathcal{Q}$ is the space of functions $f \in L^{1}(d w)$ which admit a representation of the form

$$
f(\mathbf{x})=\sum_{j=1}^{\infty} c_{j} a_{j}(\mathbf{x})
$$

where $c_{j} \in \mathbb{C}$ and $a_{j}$ are atoms for the Hardy space $H_{\mathcal{Q}}^{1, \text { at }}$ such that $\sum_{j=1}^{\infty}\left|c_{j}\right|<\infty$. The space $H_{\mathcal{Q}}^{1 \text { at }}$ is a Banach space with the norm

$$
\|f\|_{H_{\mathcal{Q}}^{1, \text { at }}}=\inf \left\{\sum_{j=1}^{\infty}\left|c_{j}\right|: f(\mathbf{x})=\sum_{j=1}^{\infty} c_{j} a_{j}(\mathbf{x}) \text { and } a_{j} \text { are } H_{\mathcal{Q}}^{1, \text { at }} \text { atoms }\right\} .
$$

Inspired by [18], we consider the following two additional conditions on $\mathcal{Q}$ and $V$ :

$$
\begin{aligned}
& (\exists C, \delta>0)\left(\forall \mathbf{x} \in \mathbb{R}^{N}, Q \in \mathcal{Q}, t \leq d(Q)^{2}\right) \int_{0}^{2 t} \int_{Q^{* * *}} V(\mathbf{y}) \mathcal{G}_{2 s / c}(\mathbf{x}, \mathbf{y}) d w(\mathbf{y}) d s \\
& \quad \leq C\left(\frac{t}{d(Q)^{2}}\right)^{\delta},
\end{aligned}
$$

where $c>0$ is the constant from Theorem 2,

$$
(\exists C, \varepsilon>0)(\forall Q \in \mathcal{Q}, s \in \mathbb{N}) \sup _{\mathbf{y} \in Q^{* * * *}} \int_{\mathbb{R}^{N}} k_{2^{s} d(Q)^{2}}(\mathbf{x}, \mathbf{y}) d w(\mathbf{x}) \leq C s^{-1-\varepsilon}
$$

The next theorem is one of the main result of the paper. We provide its proof in Sect. 9.

Theorem 7 Assume that $V \in L_{\mathrm{loc}}^{2}(d w)$ and the conditions (F), (D), and (K) hold for $V$ and a covering $\mathcal{Q}$ (let us remind that $V$ is not necessary in a reverse Hölder class). There is a constant $C>0$ such that for all $f \in L^{1}(d w)$ we have

$$
C^{-1}\|f\|_{H_{\mathcal{Q}}^{1, \text { at }}} \leq\|f\|_{H_{L}^{1}} \leq C\|f\|_{H_{\mathcal{Q}}^{1, \text { at }}} \text {. }
$$

It Sect. 10 we elaborate that the conditions (F), (D), and (K) hold for potentials $V$ satisfying the reverse Hölder inequality with $q>\max \left(1, \frac{\mathbf{N}}{2}\right)$ and the associated 
collection of cubes (48), so, as a corollary, we obtain the following theorem, which is our third main result.

Theorem 8 Assume that $V \in \mathrm{RH}^{q}(d w)$, where $q>\max \left(1, \frac{\mathbf{N}}{2}\right)$, and $V \geq 0$. There is a constant $C>0$ such that for all $f \in L^{1}(d w)$ we have

$$
C^{-1}\|f\|_{H_{\mathcal{Q}}^{1, \text { at }}} \leq\|f\|_{H_{L}^{1}} \leq C\|f\|_{H_{\mathcal{Q}}^{1, \text { at }}}
$$

where $\mathcal{Q}$ is the collection of cubes defined in (48).

We want to emphasize that the Fefferman-Phong inequality (53) is used for proving the condition (D).

\section{Local Hardy Spaces in the Dunkl Setting}

The following two definitions are inspired by [23] (see also [24]). Recall that $\left\{H_{t}\right\}_{t \geq 0}$ denotes the Dunkl heat semigroup (see (18)).

Definition 4 Let $T>0$ and $f \in L^{1}(d w)$. We say that $f$ belongs to the local Hardy space $H_{\mathrm{loc}, T}^{1}$ associated with the Dunkl Laplacian if and only if

$$
f_{\mathrm{loc}, T}^{*}(\mathbf{x})=\sup _{0<t \leq T^{2}}\left|H_{t} f(\mathbf{x})\right|
$$

belongs to $L^{1}(d w)$. The norm in the space is given by

$$
\|f\|_{H_{\mathrm{loc}, T}^{1}}=\left\|f_{\mathrm{loc}, T}^{*}\right\|_{L^{1}(d w)} \cdot
$$

Definition 5 Let $T>0$. A function $a(\mathbf{x})$ is called an atom for the local Hardy space $H_{\mathrm{loc}, T}^{1, \text { at }}$ if there is a ball $B(\mathbf{x}, r)$ such that
(A) $\operatorname{supp} a \subseteq B(\mathbf{x}, r)$,
(B) $\sup _{\mathbf{y} \in \mathbb{R}^{N}}|a(\mathbf{y})| \leq w(B(\mathbf{x}, r))^{-1}$,
(C) If $r<T$, then $\int_{\mathbb{R}^{N}} a(\mathbf{x}) d w(\mathbf{x})=0$.

A function $f$ belongs to the local Hardy space $H_{\mathrm{loc}, T}^{1, \text { at }}$ if there are $c_{j} \in \mathbb{C}$ and atoms $a_{j}$ for $H_{\mathrm{loc}, T}^{1, \text { at }}$ such that $\sum_{j=1}^{\infty}\left|c_{j}\right|<\infty$,

$$
f=\sum_{j=1}^{\infty} c_{j} a_{j} .
$$

In this case, set $\|f\|_{H_{\mathrm{loc}, T}^{1, \text { at }}}=\inf \left\{\sum_{j=1}^{\infty}\left|c_{j}\right|\right\}$, where the infimum is taken over all representations (82).

The following proposition was proved in [24] and its proof follows the pattern from [23]. 
Proposition 4 The spaces $H_{\mathrm{loc}, T}^{1, \mathrm{at}}$ and $H_{\mathrm{loc}, T}^{1}$ coincide and there is a constant $C>0$ independent of $T$ such that for all $f \in L^{1}(d w)$ we have

$$
C^{-1}\|f\|_{H_{\mathrm{loc}, T}^{1}} \leq\|f\|_{H_{\mathrm{loc}, T}^{1, \text { at }}} \leq C\|f\|_{H_{\mathrm{loc}, T}^{1}} .
$$

Moreover, there exists a constant $C>0$ such that for any $T>0$ if $f \in H_{\mathrm{loc}, T}^{1, \text { at }}$ and supp $f \subseteq B\left(\mathbf{y}_{0}, T\right)$, then there are $H_{\mathrm{loc}, T}^{1, \text { at }}$ atoms $a_{j}$ such that $\operatorname{supp} a_{j} \subseteq B\left(\mathbf{y}_{0}, 4 T\right)$ and

$$
f=\sum_{j=1}^{\infty} c_{j} a_{j}, \quad \sum_{j=1}^{\infty}\left|c_{j}\right| \leq C\|f\|_{H_{\mathrm{loc}, T}^{1, \text { at }}}
$$

\section{Auxiliary Lemmas}

Lemmas in this section are inspired by [18]. It turns out that the presence of the factor " $\left(1+\frac{\|\mathbf{x}-\mathbf{y}\|^{2}}{t}\right)^{-1}$ " in the estimate from Theorem 2 is crucial in the proof of Theorem 7 and its proper usage is the main difficulty and difference between the proofs here and in [18]. Let $\left\{\phi_{Q}\right\}_{Q \in \mathcal{Q}}$ be a smooth resolution of identity associated with the collection $\mathcal{Q}$ (see Definition 1). The existence of $\left\{\phi_{Q}\right\}_{Q \in \mathcal{Q}}$ is guaranteed by $(\mathrm{F})$.

Lemma 13 Assume that $V \in L_{\mathrm{loc}}^{2}(d w), V \geq 0$. There is a constant $C>0$ such that for all $Q \in \mathcal{Q}$ and $f \in L^{1}(d w)$ we have

$$
\begin{aligned}
& \int_{\mathbb{R}^{N} \backslash Q^{* *}} \sup _{0<t \leq d(Q)^{2}}\left|H_{t}\left(\phi_{Q} f\right)(\mathbf{x})\right| d w(\mathbf{x}) \leq C\left\|\phi_{Q} f\right\|_{L^{1}(d w)}, \\
& \int_{\mathbb{R}^{N} \backslash Q^{* *}} \sup _{0<t \leq d(Q)^{2}}\left|K_{t}\left(\phi_{Q} f\right)(\mathbf{x})\right| d w(\mathbf{x}) \leq C\left\|\phi_{Q} f\right\|_{L^{1}(d w)} .
\end{aligned}
$$

Proof We will prove just (84), thanks to (26) the proof of (85) is the same. We have

$$
\begin{aligned}
& \int_{\mathbb{R}^{N} \backslash Q^{* *}} \sup _{0<t \leq d(Q)^{2}}\left|H_{t}\left(\phi_{Q} f\right)(\mathbf{x})\right| d w(\mathbf{x}) \\
& \left.\leq \sum_{j=0}^{\infty} \int_{\mathbb{R}^{N} \backslash Q^{* *} 2^{-j-1} d(Q)^{2}<t \leq 2^{-j} d(Q)^{2}} \sup _{Q^{*}} h_{t}(\mathbf{x}, \mathbf{y})\left|\left(\phi_{Q} f\right)(\mathbf{y})\right| d w(\mathbf{y})\right) d w(\mathbf{x}) .
\end{aligned}
$$

Thanks to Theorem 2 and the fact that for $\mathbf{x} \in \mathbb{R}^{N} \backslash Q^{* *}$ and $\mathbf{y} \in Q^{*}$ we have $\|\mathbf{x}-\mathbf{y}\| \geq d(Q)$, so we obtain

$$
\left.\int_{\mathbb{R}^{N} \backslash Q^{* *} 2^{-j-1} d(Q)^{2}<t \leq 2^{-j} d(Q)^{2}} \sup _{Q^{*}} h_{t}(\mathbf{x}, \mathbf{y})\left|\left(\phi_{Q} f\right)(\mathbf{y})\right| d w(\mathbf{y})\right) d w(\mathbf{x})
$$




$$
\begin{aligned}
\leq & C \frac{2^{-j} d(Q)^{2}}{d(Q)^{2}} \int_{Q^{*}}\left|\left(\phi_{Q} f\right)(\mathbf{y})\right| \int_{\mathbb{R}^{N} \backslash Q^{* *}} \frac{1}{w\left(B\left(\mathbf{x}, 2^{-j / 2} d(Q)\right)\right)} e^{-c d(\mathbf{x}, \mathbf{y})^{2} /\left(2^{-j} d(Q)^{2}\right)} \\
& d w(\mathbf{x}) d w(\mathbf{y}) \\
\leq & C^{\prime} 2^{-j}\left\|\phi_{Q} f\right\|_{L^{1}(d w)} .
\end{aligned}
$$

The latest estimate together with (86) implies the claim.

Corollary 3 Assume that $V \in L_{\text {loc }}^{2}(d w), V \geq 0$. There is a constant $C>0$ such that for every $Q \in \mathcal{Q}$ and $f \in L^{1}(d w)$ we have

$$
\left\|\phi_{Q} f\right\|_{H_{\mathrm{loc}, d(Q)}^{1}} \leq C\left\|\sup _{0<t \leq d(Q)^{2}}\left|H_{t}\left(\phi_{Q} f\right)\right|\right\|_{L^{1}\left(Q^{* *}, d w\right)}+C\left\|\phi_{Q} f\right\|_{L^{1}(d w)} .
$$

For $Q \in \mathcal{Q}$ we define

$$
\begin{aligned}
& \mathcal{Q}^{\prime}(Q)=\left\{Q^{\prime} \in \mathcal{Q}: Q^{* * *} \cap\left(Q^{\prime}\right)^{* * *} \neq \emptyset\right\}, \\
& \mathcal{Q}^{\prime \prime}(Q)=\left\{Q^{\prime \prime} \in \mathcal{Q}: Q^{* * *} \cap\left(Q^{\prime \prime}\right)^{* * *}=\emptyset\right\} .
\end{aligned}
$$

Lemma 14 Assume that $V \in L_{\mathrm{loc}}^{2}(d w), V \geq 0$. There is a constant $C>0$ such that for every $Q \in \mathcal{Q}$ and $f \in L^{1}\left(\mathbb{R}^{N}\right)$ we have

$$
\left\|\sup _{0<t \leq d(Q)^{2}}\left|K_{t}\left(\phi_{Q} g\right)-\phi_{Q} K_{t}(g)\right|\right\|_{L^{1}\left(Q^{* *}, d w\right)} \leq C \sum_{Q^{\prime} \in \mathcal{Q}^{\prime}(Q)}\left\|\phi_{Q^{\prime}} f\right\|_{L^{1}(d w)},(90)
$$

where $g=\sum_{Q^{\prime} \in \mathcal{Q}^{\prime}(Q)} \phi_{Q^{\prime}} f$.

Proof Thanks to (59), then Theorem 2 together with (26) and (10) we get

$$
\begin{aligned}
& \sup _{0<t \leq d(Q)^{2}}\left|K_{t}\left(\phi_{Q} g\right)(\mathbf{x})-\phi_{Q}(\mathbf{x}) K_{t} g(\mathbf{x})\right| \\
& =\sup _{0<t \leq d(Q)^{2}}\left|\int_{\mathbb{R}^{N}}\left(\phi_{Q}(\mathbf{y})-\phi_{Q}(\mathbf{x})\right) k_{t}(\mathbf{x}, \mathbf{y}) g(\mathbf{y}) d w(\mathbf{y})\right| \\
& \leq C \sup _{0<t \leq d(Q)^{2}} \int_{\mathbb{R}^{N}} \frac{\|\mathbf{x}-\mathbf{y}\|}{d(Q)} k_{t}(\mathbf{x}, \mathbf{y})|g(\mathbf{y})| d w(\mathbf{y}) \\
& \leq C \sup _{0<t \leq d(Q)^{2}} \int_{\mathbb{R}^{N}} \frac{\|\mathbf{x}-\mathbf{y}\|}{d(Q)} \frac{\sqrt{t}}{\|\mathbf{x}-\mathbf{y}\|} \frac{1}{w(B(\mathbf{y}, \sqrt{t}))} e^{-c d(\mathbf{x}, \mathbf{y})^{2} / t}|g(\mathbf{y})| d w(\mathbf{y}) \\
& \leq C \sum_{j=0}^{\infty} 2^{2^{-j-1} d(Q)^{2}<t \leq 2^{-j} d(Q)^{2}} \int_{\mathbb{R}^{N}} \frac{\sqrt{t}}{d(Q)} \frac{1}{w(B(\mathbf{y}, \sqrt{t}))} e^{-c d(\mathbf{x}, \mathbf{y})^{2} / t}|g(\mathbf{y})| d w(\mathbf{y}) \\
& \leq C \sum_{j=0}^{\infty} \int_{\mathbb{R}^{N}} \frac{2^{-j / 2} d(Q)}{d(Q)} \frac{1}{w\left(B\left(\mathbf{y}, 2^{-j / 2} d(Q)\right)\right)} e^{-c d(\mathbf{x}, \mathbf{y})^{2} /\left(2^{-j} d(Q)^{2}\right)}|g(\mathbf{y})| d w(\mathbf{y}) .
\end{aligned}
$$


Consequently, by the Fubini theorem,

$\left\|\sup _{0<t \leq d(Q)^{2}}\left|K_{t}\left(\phi_{Q} g\right)(\mathbf{x})-\phi_{Q}(\mathbf{x}) K_{t} g(\mathbf{x})\right|\right\|_{L^{1}(d w(\mathbf{x}))} \leq C \sum_{j=0}^{\infty} 2^{-j / 2}\|g\|_{L^{1}(d w)} \leq C\|g\|_{L^{1}}$.

Lemma 15 Assume that $\mathcal{Q}$ and $V \in L_{\text {loc }}^{2}(d w), V \geq 0$, satisfy the condition (D). Then there is a constant $C>0$ such that for all $f \in L^{1}(d w)$ we have

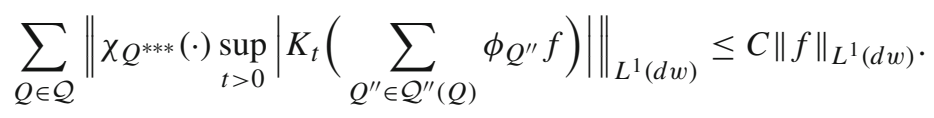

Proof Let us denote the left-hand side of (91) by $S$. Then, by (F), we get

$$
\begin{aligned}
& S \leq \sum_{Q \in \mathcal{Q}} \sum_{Q^{\prime \prime} \in \mathcal{Q}^{\prime \prime}(Q)}\left\|\chi_{Q^{* * *}(\cdot)} \sup _{t>0}\left(K_{t}\left|\phi_{Q^{\prime \prime}} f\right|\right)\right\|_{L^{1}(d w)} \\
& \leq \sum_{Q^{\prime \prime} \in \mathcal{Q}} \sum_{Q \in \mathcal{Q}^{\prime \prime}\left(Q^{\prime \prime}\right)}\left\|\chi Q^{* * *}(\cdot) \sup _{t>0}\left(K_{t}\left|\phi_{Q^{\prime \prime}} f\right|\right)\right\|_{L^{1}(d w)} \\
& \leq C \sum_{Q^{\prime \prime} \in \mathcal{Q}}\left\|\sup _{t>0}\left(K_{t}\left|\phi_{Q^{\prime \prime}} f\right|\right)\right\|_{L^{1}\left(\left(\left(Q^{\prime \prime}\right)^{* *}\right)^{c}, d w\right)} \\
& \leq C \sum_{Q^{\prime \prime} \in \mathcal{Q}}\left\|\sup _{0<t<d\left(Q^{\prime \prime}\right)^{2}}\left(K_{t}\left|\phi_{Q^{\prime \prime}} f\right|\right)\right\|_{L^{1}\left(\left(\left(Q^{\prime \prime}\right)^{* *}\right)^{c}, d w\right)} \\
& +\sum_{j=0}^{\infty} \sum_{Q^{\prime \prime} \in \mathcal{Q}}\left\|\sup _{2^{j} d\left(Q^{\prime \prime}\right)^{2} \leq t<2^{j+1} d\left(Q^{\prime \prime}\right)^{2}}\left(K_{t}\left|\phi_{Q^{\prime \prime}} f\right|\right)\right\|_{L^{1}\left(\left(\left(Q^{\prime \prime}\right)^{* *}\right)^{c}, d w\right)}=: S_{1}+S_{2} \text {. }
\end{aligned}
$$

The estimate $S_{1} \leq C\|f\|_{L^{1}(d w)}$ follows from (85) and (F). Furthermore, by the semigroup property and Theorem 2 together with (26), for $2^{j} d(Q)^{2} \leq t<2^{j+1} d(Q)^{2}$, we have

$$
\begin{aligned}
& \int_{\mathbb{R}^{N}} k_{t}(\mathbf{x}, \mathbf{y})\left|\left(\phi_{Q^{\prime \prime}} f\right)(\mathbf{y})\right| d w(\mathbf{y}) \\
& \quad=\int_{\mathbb{R}^{N}} \int_{\mathbb{R}^{N}} k_{t-2^{j-1} d\left(Q^{\prime \prime}\right)^{2}(\mathbf{x}, \mathbf{z}) k_{2^{j-1}} d\left(Q^{\prime \prime}\right)^{2}(\mathbf{z}, \mathbf{y}) d w(\mathbf{z})\left|\left(\phi_{Q^{\prime \prime}} f\right)(\mathbf{y})\right| d w(\mathbf{y})} \\
& \quad \leq C \int_{\mathbb{R}^{N}} \int_{\mathbb{R}^{N}} \frac{1}{w\left(B\left(\mathbf{x}, 2^{j / 2} d\left(Q^{\prime \prime}\right)\right)\right)} e^{\frac{-c d(\mathbf{x}, \mathbf{z})^{2}}{2^{j+1} d\left(Q^{\prime \prime}\right)^{2}}} k_{2^{j-1}} d\left(Q^{\prime \prime}\right)^{2}(\mathbf{z}, \mathbf{y}) d w(\mathbf{z})\left|\left(\phi_{Q^{\prime \prime}} f\right)(\mathbf{y})\right| d w(\mathbf{y}) .
\end{aligned}
$$

Integrating over the set $\left(\left(Q^{\prime \prime}\right)^{* *}\right)^{c}$ with respect to the $\mathbf{x}$-variable, we obtain

$$
\int_{\left(\left(Q^{\prime \prime}\right)^{* *}\right)^{c}}\left(\sup _{2^{j} d\left(Q^{\prime \prime}\right)^{2} \leq t<2^{j+1} d\left(Q^{\prime \prime}\right)^{2}} \int_{\mathbb{R}^{N}} k_{t}(\mathbf{x}, \mathbf{y})\left|\left(\phi_{Q^{\prime \prime}} f\right)(\mathbf{y})\right| d w(\mathbf{y})\right) d w(\mathbf{x})
$$




$$
\leq C \int_{\mathbb{R}^{N}}\left|\left(\phi_{Q^{\prime \prime}} f\right)(\mathbf{y})\right| \int_{\mathbb{R}^{N}} k_{2^{j-1} d\left(Q^{\prime \prime}\right)^{2}}(\mathbf{z}, \mathbf{y}) d w(\mathbf{z}) d w(\mathbf{y}) .
$$

Consequently, by (D) and (85), we get

$$
S_{2} \leq C \sum_{Q^{\prime \prime} \in \mathcal{Q}}\left\|\phi_{Q^{\prime \prime}} f\right\|_{L^{1}(d w)}+C \sum_{j=1}^{\infty} \sum_{Q^{\prime \prime} \in \mathcal{Q}} j^{-1-\varepsilon}\left\|\phi_{Q^{\prime \prime}} f\right\|_{L^{1}(d w)} \leq C\|f\|_{L^{1}(d w)}
$$

Lemma 16 Assume that $V \in L_{\mathrm{loc}}^{2}(d w), V \geq 0$. For all $f \in L^{1}(d w)$ we have

$$
\int_{\mathbb{R}^{N}} \int_{0}^{\infty} V(\mathbf{x}) K_{s}|f|(\mathbf{x}) d s d w(\mathbf{x}) \leq\|f\|_{L^{1}(d w)} .
$$

Proof The lemma is well-known. We provide the proof for the sake of completeness. By the perturbation formula we have

$$
H_{t}|f|(\mathbf{x})-K_{t}|f|(\mathbf{x})=\int_{0}^{t}\left(H_{t-s} V K_{s}\right)|f|(\mathbf{x}) d s,
$$

so, by (26), we obtain

$$
\int_{0}^{t} \int_{\mathbb{R}^{N}} h_{t-s}(\mathbf{x}, \mathbf{y}) V K_{s}|f|(\mathbf{y}) d w(\mathbf{y}) d s \leq \int_{\mathbb{R}^{N}} h_{t}(\mathbf{x}, \mathbf{y})|f|(\mathbf{y}) d w(\mathbf{y}) .
$$

Integrating (93) with respect to the $\mathbf{x}$-variable, using the Fubini theorem and the fact that for all $t>0$ we have $\int_{\mathbb{R}^{N}} h_{t}(\mathbf{x}, \mathbf{y}) d w(\mathbf{x})=1$ (see (20)), we get

$$
\int_{0}^{t} \int_{\mathbb{R}^{N}} V(\mathbf{y}) K_{s}|f|(\mathbf{y}) d w(\mathbf{y}) d s \leq\|f\|_{L^{1}(d w)} .
$$

Letting $t \rightarrow \infty$ we obtain the lemma.

Lemma 17 Assume that $\mathcal{Q}$ and $V \in L_{\text {loc }}^{2}(d w), V \geq 0$, satisfy $(\mathrm{K})$. There is a constant $C>0$ such that for all $Q \in \mathcal{Q}$ and $f \in L^{1}(d w)$ we have

$$
\left\|\sup _{0<t \leq d(Q)^{2}}\left|\left(H_{t}-K_{t}\right)\left(\phi_{Q} f\right)\right|\right\|_{L^{1}(d w)} \leq C\left\|\phi_{Q} f\right\|_{L^{1}(d w)} .
$$

Proof Thanks to (84) and (85) it is enough to estimate

$$
\left\|\sup _{0<t \leq d(Q)^{2}}\left|\left(H_{t}-K_{t}\right)\left(\phi_{Q} f\right)\right|\right\|_{L^{1}\left(Q^{* *}, d w\right)} .
$$


By the perturbation formula we write

$$
\begin{aligned}
H_{t}\left(\phi_{Q} f\right)(\mathbf{x})-K_{t}\left(\phi_{Q} f\right)(\mathbf{x})= & \int_{0}^{t} \int_{\mathbb{R}^{N}} h_{t-s}(\mathbf{x}, \mathbf{y}) V(\mathbf{y}) K_{S}\left(\phi_{Q} f\right)(\mathbf{y}) d w(\mathbf{y}) d s \\
= & \int_{0}^{t} \int_{\mathbb{R}^{N}} h_{t-s}(\mathbf{x}, \mathbf{y}) V_{1}(\mathbf{y}) K_{s}\left(\phi_{Q} f\right)(\mathbf{y}) d w(\mathbf{y}) d s \\
& +\int_{0}^{t} \int_{\mathbb{R}^{N}} h_{t-s}(\mathbf{x}, \mathbf{y}) V_{2}(\mathbf{y}) K_{s}\left(\phi_{Q} f\right)(\mathbf{y}) d w(\mathbf{y}) d s
\end{aligned}
$$

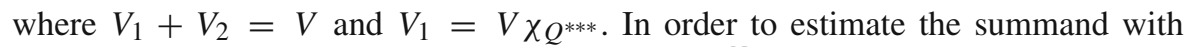
$V_{2}$, we use Theorem 2 and the fact that for $\mathbf{y} \in \mathbb{R}^{N} \backslash Q^{* * *}$ and $\mathbf{x} \in Q^{* *}$ we have $\|\mathbf{x}-\mathbf{y}\| \geq d(Q)$, so

$$
\begin{aligned}
& \sup _{0<t \leq d(Q)^{2}}\left|\int_{0}^{t} \int_{\mathbb{R}^{N}} h_{t-s}(\mathbf{x}, \mathbf{y}) V_{2}(\mathbf{y}) K_{S}\left(\phi_{Q} f\right)(\mathbf{y}) d w(\mathbf{y}) d s\right| \\
& \leq \sum_{j=02-j-1 d(Q)^{2}<t \leq 2^{-j} d(Q)^{2}}^{\infty} \int_{0}^{t} \int_{\mathbb{R}^{N}} h_{t-s}(\mathbf{x}, \mathbf{y}) V_{2}(\mathbf{y}) K_{S}\left(\left|\phi_{Q} f\right|\right)(\mathbf{y}) d w(\mathbf{y}) d s \\
& =\sum_{j=02-j-1 d(Q)^{2}<t \leq 2^{-j} d(Q)^{2}}^{\infty} \sum_{\ell=0}^{\infty} \int_{t-2^{-\ell} t}^{t-2^{-\ell-1} t} \int_{\mathbb{R}^{N}} h_{t-s}(\mathbf{x}, \mathbf{y}) V_{2}(\mathbf{y}) K_{S}\left(\left|\phi_{Q} f\right|\right)(\mathbf{y}) d w(\mathbf{y}) d s \\
& \leq C \sum_{j, \ell=02-j-1 d(Q)^{2}<t \leq 2^{-j} d(Q)^{2}}^{\infty} \int_{t-2^{-\ell} t}^{t-2^{-\ell-1} t} \int_{\mathbb{R}^{N}} \frac{t-s}{\|\mathbf{x}-\mathbf{y}\|^{2}} \frac{1}{w(B(\mathbf{x}, \sqrt{t-s}))} \\
& \quad e^{-c d(\mathbf{x}, \mathbf{y})^{2} /(t-s)} V_{2}(\mathbf{y}) K_{S}|(\phi Q f)|(\mathbf{y}) d w(\mathbf{y}) d s \\
& \leq C \sum_{j, \ell=0}^{\infty} \int_{0}^{\infty} \int_{\mathbb{R}^{N}}^{\infty} \frac{2^{-j-\ell} d(Q)^{2}}{d(Q)^{2}} \frac{1}{w\left(B\left(\mathbf{x}, 2^{-(j+\ell) / 2} d(Q)\right)\right)} e^{-\frac{c d(\mathbf{x}, \mathbf{y})^{2}}{\left(2^{-j-\ell} d(Q)^{2}\right)}} \\
& \quad V(\mathbf{y}) K_{S}\left|\left(\phi_{Q} f\right)\right|(\mathbf{y}) d w(\mathbf{y}) d s .
\end{aligned}
$$

Therefore, by the Fubini theorem and (92), we obtain

$$
\begin{aligned}
& \left\|\sup _{0<t \leq d(Q)^{2}} \int_{0}^{t} \int_{\mathbb{R}^{N}} h_{t-s}(\mathbf{x}, \mathbf{y}) V_{2}(\mathbf{y}) K_{s}\left(\phi_{Q} f\right)(\mathbf{y}) d w(\mathbf{y}) d s\right\|_{L^{1}\left(Q^{* *}, d w\right)} \\
& \leq C \sum_{j, l=0}^{\infty} 2^{-j-l} \int_{0}^{\infty} \int_{\mathbb{R}^{N}} V(\mathbf{y}) K_{s}\left|\left(\phi_{Q} f\right)\right|(\mathbf{y}) d w(\mathbf{y}) d s \leq C\left\|\phi_{Q} f\right\|_{L^{1}(d w)}
\end{aligned}
$$

In order to estimate the summand in (95) containing $V_{1}$, we write 


$$
\int_{0}^{t} \int_{\mathbb{R}^{N}} h_{t-s}(\mathbf{x}, \mathbf{y}) V_{1}(\mathbf{y}) K_{S}\left(\phi_{Q} f\right)(\mathbf{y}) d w(\mathbf{y}) d s=\int_{0}^{t / 2} \ldots+\int_{t / 2}^{t} \ldots=: I_{t}(\mathbf{x})+J_{t}(\mathbf{x}) .
$$

Clearly, by Theorem 2 and the Fubini theorem, we get

$$
\begin{aligned}
& \left\|\sup _{0<t \leq d(Q)^{2}}\left|I_{t}(\mathbf{x})\right|\right\|_{L^{1}(d w(\mathbf{x}))} \leq \sum_{j=0}^{\infty}\left\|_{2^{-j-1} d(Q)^{2}<t \leq 2^{-j} d(Q)^{2}} \sup _{t}(\mathbf{x}) \mid\right\|_{L^{1}(d w(\mathbf{x}))} \\
& \leq C \sum_{j=0}^{\infty} \int_{\mathbb{R}^{N}} \int_{0}^{2^{-j} d(Q)^{2}} \int_{\mathbb{R}^{N}} \frac{1}{w\left(B\left(\mathbf{y}, 2^{-j / 2} d(Q)\right)\right)} e^{\frac{-c d(\mathbf{x}, \mathbf{y})^{2}}{2^{-j} d(Q)^{2}}} V_{1}(\mathbf{y}) K_{s}\left|\left(\phi_{Q} f\right)\right|(\mathbf{y}) d w(\mathbf{y}) d s d w(\mathbf{x}) \\
& \leq C \sum_{j=0}^{\infty} \int_{0}^{2^{-j} d(Q)^{2}} \int_{\mathbb{R}^{N}} V_{1}(\mathbf{y}) K_{S}\left|\left(\phi_{Q} f\right)\right|(\mathbf{y}) d w(\mathbf{y}) d s \\
& =C \sum_{j=0}^{\infty} \int_{0}^{2^{-j} d(Q)^{2}} \int_{Q^{* * *}} V(\mathbf{y}) \int_{\mathbb{R}^{N}} k_{s}(\mathbf{y}, \mathbf{z})\left|\left(\phi_{Q} f\right)(\mathbf{z})\right| d w(\mathbf{z}) d w(\mathbf{y}) d s \\
& \leq C^{\prime} \sum_{j=0}^{\infty} \int_{\mathbb{R}^{N}}\left|\left(\phi_{Q} f\right)(\mathbf{z})\right|\left(\int_{0}^{2^{-j} d(Q)^{2}} \int_{Q^{* * *}} V(\mathbf{y}) \mathcal{G}_{s / c}(\mathbf{y}, \mathbf{z}) d w(\mathbf{y}) d s\right) d w(\mathbf{z}),
\end{aligned}
$$

where in the last step we have used (26) and Theorem 2. Consequently, by assumption $(\mathrm{K})$, we get

$$
\left\|\sup _{0<t \leq d(Q)^{2}}\left|I_{t}(\mathbf{x})\right|\right\|_{L^{1}(d w(\mathbf{x}))} \leq C \sum_{j=0}^{\infty} 2^{-j \delta}\left\|\left(\phi_{Q} f\right)\right\|_{L^{1}(d w)} \leq C\left\|\left(\phi_{Q} f\right)\right\|_{L^{1}(d w)} .
$$

Similarly, we write

$$
\left\|\sup _{0<t \leq d(Q)^{2}}\left|J_{t}(\mathbf{x})\right|\right\|_{L^{1}(d w(\mathbf{x}))} \leq \sum_{j=0}^{\infty}\left\|\sup _{2^{-j-1} d(Q)^{2}<t \leq 2^{-j} d(Q)^{2}}\left|J_{t}(\mathbf{x})\right|\right\|_{L^{1}(d w(\mathbf{x}))} .
$$

Then, by changing of variables, we have

$$
\left|J_{t}(\mathbf{x})\right|=\int_{\mathbb{R}^{N}} \int_{0}^{t / 2} \int_{\mathbb{R}^{N}} h_{S}(\mathbf{x}, \mathbf{y}) V_{1}(\mathbf{y}) k_{t-s}(\mathbf{y}, \mathbf{z})\left(\left|\phi_{Q} f\right|\right)(\mathbf{z}) d w(\mathbf{y}) d s d w(\mathbf{z}) .
$$

Applying Theorem 2 and (26), we get

$$
\begin{aligned}
& \sup _{2^{-j-1} d(Q)^{2}<t \leq 2^{-j} d(Q)^{2}}\left|J_{t}(\mathbf{x})\right| \\
& \leq C \int_{\mathbb{R}^{N}} \int_{0}^{2^{-j-1} d(Q)^{2}} \int_{\mathbb{R}^{N}} \mathcal{G}_{S / c}(\mathbf{x}, \mathbf{y}) V_{1}(\mathbf{y}) \mathcal{G}_{2^{-j}} d(Q)^{2} / c \\
&
\end{aligned}
$$


Note that for $s \leq \frac{t}{2} \leq 2^{-j-1} d(Q)^{2}$ we have

$$
\begin{aligned}
e^{-c d(\mathbf{x}, \mathbf{y})^{2} / s} e^{-c d(\mathbf{y}, \mathbf{z})^{2} /\left(2^{-j} d(Q)^{2}\right)} & \leq e^{-c d(\mathbf{x}, \mathbf{y})^{2} /(2 s)} e^{-c d(\mathbf{x}, \mathbf{y})^{2} /\left(2^{-j} d(Q)^{2}\right)} e^{-c d(\mathbf{y}, \mathbf{z})^{2} /\left(2^{-j} d(Q)^{2}\right)} \\
& \leq e^{-c d(\mathbf{x}, \mathbf{y})^{2} /(2 s)} e^{-c d(\mathbf{x}, \mathbf{z})^{2} /\left(2^{-j+1} d(Q)^{2}\right)}
\end{aligned}
$$

so (97) and the doubling property of $w$ lead us to

$$
\begin{aligned}
& \sup _{2^{-j-1} d(Q)^{2}<t \leq 2^{-j} d(Q)^{2}}\left|J_{t}(\mathbf{x})\right| \\
& \leq \int_{\mathbb{R}^{N}} \int_{0}^{2^{-j-1} d(Q)^{2}} \int_{\mathbb{R}^{N}} \mathcal{G}_{2 s / c}(\mathbf{x}, \mathbf{y}) V_{1}(\mathbf{y}) \frac{1}{w\left(B\left(\mathbf{z}, 2^{-j / 2} d(Q)\right)\right)} e^{-\frac{c d(\mathbf{x}, \mathbf{z})^{2}}{2^{-j+1} d(Q)^{2}}} \\
& \quad\left(\left|\phi_{Q} f\right|\right)(\mathbf{z}) d w(\mathbf{y}) d s d w(\mathbf{z}) .
\end{aligned}
$$

Furthermore, by the assumption (K), we get

$$
\begin{aligned}
& \sup _{2^{-j-1} d(Q)^{2}<t \leq 2^{-j} d(Q)^{2}}\left|J_{t}(\mathbf{x})\right| \\
& \leq C 2^{-j \delta} \int_{\mathbb{R}^{N}} \frac{1}{w\left(B\left(\mathbf{z}, 2^{-j / 2} d(Q)\right)\right)} e^{-\frac{c d(\mathbf{x}, \mathbf{z})^{2}}{2^{-j+1} d(Q)^{2}}}\left(\left|\phi_{Q} f\right|\right)(\mathbf{z}) d w(\mathbf{z}) .
\end{aligned}
$$

Finally, integrating (98) with respect to $\mathbf{x}$-variable and taking (96) into account we see that the proof is complete.

\section{Proof of Theorem 7}

The proof is based on the proof of [18, Theorem 2.2]. Let us remind that $\left\{\phi_{Q}\right\}_{Q \in \mathcal{Q}}$ is the smooth resolution of identity associated with the covering $\mathcal{Q}$ (see Definition 1 ).

\subsection{Proof of the Inequality $C^{-1}\|f\|_{H_{\mathcal{Q}}^{1, \text { at }}} \leq\|f\|_{H_{L}^{1}}$}

Thanks to Corollary 3 and Lemma 17 we have

$$
\begin{aligned}
& \sum_{Q \in \mathcal{Q}}\left\|\phi_{Q} f\right\|_{H_{\mathrm{loc}, d(Q)}^{1}} \leq C \sum_{Q \in \mathcal{Q}}\left\|\sup _{0<t \leq d(Q)^{2}}\left|H_{t}\left(\phi_{Q} f\right)\right|\right\|_{L^{1}\left(Q^{* *}, d w\right)}+C\|f\|_{L^{1}(d w)} \\
& \leq C \sum_{Q \in \mathcal{Q}}\left\|\sup _{0<t \leq d(Q)^{2}}\left|\left(H_{t}-K_{t}\right)\left(\phi_{Q} f\right)\right|\right\|_{L^{1}\left(Q^{* *}, d w\right)} \\
& \quad+C \sum_{Q \in \mathcal{Q}}\left\|\sup _{0<t \leq d(Q)^{2}}\left|K_{t}\left(\phi_{Q} f\right)\right|\right\|_{L^{1}\left(Q^{* *}, d w\right)} \\
& \quad+C\|f\|_{L^{1}(d w)}
\end{aligned}
$$




$$
\leq C\|f\|_{L^{1}(d w)}+C \sum_{Q \in \mathcal{Q}}\left\|\sup _{0<t \leq d(Q)^{2}}\left|K_{t}\left(\phi_{Q} f\right)\right|\right\|_{L^{1}\left(Q^{* *}, d w\right)} .
$$

Then, by Lemma 14 and (91) we get

$$
\begin{aligned}
\sum_{Q \in \mathcal{Q}} & \left\|\sup _{0<t \leq d(Q)^{2}}\left|K_{t}\left(\phi_{Q} f\right)\right|\right\|_{L^{1}\left(Q^{* *}, d w\right)} \int_{Q_{Q \in \mathcal{Q}}} \int_{Q^{* *}} \sup _{0<t \leq d(Q)^{2}}\left|K_{t}\left(\phi_{Q} \sum_{Q^{\prime} \in \mathcal{Q}^{\prime}(Q)}\left(\phi_{Q^{\prime}} f\right)\right)(\mathbf{x})\right| d w(\mathbf{x}) \\
\leq & \sum_{Q \in \mathcal{Q}} \int_{Q^{* *}} \sup _{0<t \leq d(Q)^{2}} \mid K_{t}\left(\phi_{Q} \sum_{Q^{\prime} \in \mathcal{Q}^{\prime}(Q)}\left(\phi_{Q^{\prime}} f\right)\right)(\mathbf{x}) \\
& -\phi_{Q}(\mathbf{x})\left(K_{t}\left(\sum_{Q^{\prime} \in \mathcal{Q}^{\prime}(Q)}\left(\phi_{Q^{\prime}} f\right)\right)(\mathbf{x}) \mid d w(\mathbf{x})\right. \\
& +\sum_{Q \in \mathcal{Q}} \int_{Q^{* *}} \sup _{0<t \leq d(Q)^{2}} \mid \phi_{Q}(\mathbf{x})\left(K_{t}\left(\sum_{Q^{\prime \prime} \in \mathcal{Q}^{\prime \prime}(Q)}\left(\phi_{Q^{\prime \prime}} f\right)\right)(\mathbf{x}) \mid d w(\mathbf{x})\right. \\
& +\sum_{Q \in \mathcal{Q}} \int_{Q^{* *}} \phi_{Q}(\mathbf{x}) \sup _{0<t \leq d(Q)^{2}}\left|K_{t}(f)(\mathbf{x})\right| d w(\mathbf{x}) \\
\leq & C\|f\|_{L^{1}(d w)}+\left\|\sup _{t>0}\left|K_{t} f\right|\right\|_{L^{1}(d w)} \cdot
\end{aligned}
$$

Hence, we have obtained

$$
\sum_{Q \in \mathcal{Q}}\left\|\phi_{Q} f\right\|_{H_{\mathrm{loc}, d(Q)}^{1}} \leq C\|f\|_{H_{L}^{1}}
$$

Therefore, by Proposition 4 we get

$$
\phi_{Q}(\mathbf{x}) f(\mathbf{x})=\sum_{j=0}^{\infty} c_{j, Q}(\mathbf{x}) a_{j, Q}(\mathbf{x})
$$

where $a_{j, Q}$ are atoms of local Hardy space $H_{\mathrm{loc}, d(Q)}^{1, \text { at }}$ (see Definition 5 and Proposition 4) and

$$
\sum_{Q \in \mathcal{Q}} \sum_{j=0}^{\infty}\left|c_{j, Q}\right| \leq C\|f\|_{H_{L}^{1}}
$$

Since $\operatorname{supp} \phi_{Q} f \subseteq Q^{*}$, Proposition 4 guaranteed that we may get $a_{j, Q}$ such that $\operatorname{supp} a_{j, Q} \subseteq Q^{* * * *}$. Consequently, by Definition 3 , each $a_{j, Q}$ is an atom of $H_{\mathcal{Q}}^{1 \text {, at }}$. 


\subsection{Proof of the Inequality $\|f\|_{H_{L}^{1}} \leq C\|f\|_{H_{\mathcal{Q}}^{1, \text { at }}}$}

It is enough to check if there is a constant $C>0$ such that for all atoms $a(\mathbf{x})$ of $H_{\mathcal{Q}}^{1 \text {, at }}$ we have $\|a\|_{H_{L}^{1}} \leq C$. Suppose that $a(\mathbf{x})$ is associated with a cube $Q \in \mathcal{Q}$. We write

$$
a=\sum_{Q^{\prime} \in \mathcal{Q}} \phi_{Q^{\prime}} a
$$

Thanks to (F) and the fact that $\operatorname{supp} a \subseteq Q^{* * * *}$, there is a number $M>0$ independent of $Q$ such that in (99) there are at most $M$ nonzero summands and all of them satisfy $d\left(Q^{\prime}\right) \sim d(Q)$. Let $\ell \geq 0$ be the smallest positive integer such that $d\left(Q^{\prime}\right) \geq 2^{-\ell / 2} d(Q)$ for all such a nonzero summands $\phi_{Q^{\prime}} a$ in (99). Clearly, thanks to $(\mathrm{F}), \ell$ is independent of $a$ and $Q \in \mathcal{Q}$. We write

$$
\|a\|_{H_{L}^{1}} \leq\left\|\sup _{0<t \leq 2^{-\ell} d(Q)^{2}}\left|K_{t} a\right|\right\|\left\|_{L^{1}(d w)}+\right\| \sup _{t>2^{-\ell} d(Q)^{2}}\left|K_{t} a\right| \|_{L^{1}(d w)}=: I_{1}+I_{2} .
$$

Further,

$$
I_{1} \leq\left\|\sup _{0<t \leq 2^{-\ell} d(Q)^{2}}\left|\left(K_{t}-H_{t}\right) a\right|\right\|\left\|_{L^{1}(d w)}+\right\| \sup _{0<t \leq 2^{-\ell} d(Q)^{2}}\left|H_{t} a\right| \|_{L^{1}(d w)} .
$$

Thanks to the fact that atom $a$ is, by definition, an atom for $H_{\mathrm{loc}, d(Q)}^{1}$, we have

$$
\left\|\sup _{0<t \leq 2^{-\ell} d(Q)^{2}}\left|H_{t} a\right|\right\|_{L^{1}(d w)} \leq\left\|\sup _{0<t \leq d(Q)^{2}}\left|H_{t} a\right|\right\|_{L^{1}(d w)} \leq C .
$$

Thanks to (94) and (99), we get

$$
\begin{aligned}
& \left\|\sup _{0<t \leq 2^{-\ell} d(Q)^{2}}\left|\left(K_{t}-H_{t}\right) \sum_{Q^{\prime} \in \mathcal{Q}}\left(\phi_{Q^{\prime}} a\right)\right|\right\|_{L^{1}(d w)} \\
& \leq \sum_{Q^{\prime} \in \mathcal{Q}}\left\|\sup _{0<t \leq d\left(Q^{\prime}\right)^{2}}\left|\left(K_{t}-H_{t}\right)\left(\phi_{Q^{\prime}} a\right)\right|\right\|_{L^{1}(d w)} \\
& \leq C \sum_{Q^{\prime} \in Q}\left\|\phi_{Q^{\prime}} a\right\|_{L^{1}(d w)} \leq C M\|a\|_{L^{1}(d w)} \leq C .
\end{aligned}
$$


In order to estimate $I_{2}$, we repeat the argument presented in the proof of (91). We provide details. We write

$$
I_{2} \leq \sum_{j=-\ell}^{\infty}\left\|\sup _{2^{j} d(Q)^{2}<t \leq 2^{j+1} d(Q)^{2}}\left|K_{t} a\right|\right\|_{L^{1}(d w)}
$$

By the semigroup property, Theorem 2 together with (26), for

$$
2^{j} d(Q)^{2}<t \leq 2^{j+1} d(Q)^{2}
$$

we have

$$
\begin{aligned}
& \int_{\mathbb{R}^{N}} k_{t}(\mathbf{x}, \mathbf{y})|a(\mathbf{y})| d w(\mathbf{y}) \\
& \quad=\int_{\mathbb{R}^{N}} \int_{\mathbb{R}^{N}} k_{t-2^{j-1} d(Q)^{2}(\mathbf{x}, \mathbf{z}) k_{2^{j-1}} d(Q)^{2}(\mathbf{z}, \mathbf{y}) d w(\mathbf{z})|a(\mathbf{y})| d w(\mathbf{y})} \\
& \quad \leq C \int_{\mathbb{R}^{N}} \int_{\mathbb{R}^{N}} \frac{1}{w\left(B\left(\mathbf{z}, 2^{j / 2} d(Q)\right)\right)} e^{-c d(\mathbf{x}, \mathbf{z})^{2} /\left(2^{j+1} d(Q)^{2}\right)} k_{2^{j-1} d(Q)^{2}}(\mathbf{z}, \mathbf{y}) d w(\mathbf{z})|a(\mathbf{y})| d w(\mathbf{y}) .
\end{aligned}
$$

Therefore, integrating over the $\mathbf{x}$-variable, we obtain

$$
\begin{gathered}
\int_{\mathbb{R}^{N} 2^{j} d(Q)^{2} \leq t<2^{j+1} d(Q)^{2}} \int_{\mathbb{R}^{N}} k_{t}(\mathbf{x}, \mathbf{y})|a(\mathbf{y})| d w(\mathbf{y}) d w(\mathbf{x}) \\
\leq C \int_{\mathbb{R}^{N}}|a(\mathbf{y})| \int_{\mathbb{R}^{N}} k_{2^{j-1}} d(Q)^{2}(\mathbf{z}, \mathbf{y}) d w(\mathbf{z}) d w(\mathbf{y}) .
\end{gathered}
$$

Consequently, by the condition (D) and (100), we get

$$
I_{2} \leq C\left(\ell+\sum_{j=1}^{\infty} j^{-1-\varepsilon}\right)\|a\|_{L^{1}(d w)} \leq C^{\prime}\|a\|_{L^{1}(d w)} \leq C^{\prime} .
$$

\section{Verification of Conditions (D) and (K) for potentials from the reverse Hölder class}

Let us note that the condition (F) for $V \in \mathrm{RH}^{q}(d w), q>\max \left(1, \frac{\mathbf{N}}{2}\right)$, is already checked, see Proposition 3.

\subsection{Verification of Condition (D)}

We will begin with the lemma, which holds for all $V \in L_{\mathrm{loc}}^{2}(d w)$ (not necessary $\left.V \in \mathrm{RH}^{q}(d w)\right)$. 
Lemma 18 Assume that $V \in L_{\mathrm{loc}}^{2}(d w), V \geq 0$. There is a constant $C>0$ such that for all $\mathbf{y} \in \mathbb{R}^{N}$ and $t>0$ we have

$$
\left\langle L k_{t}(\cdot, \mathbf{y}), k_{t}(\cdot, \mathbf{y})\right\rangle \leq \frac{C}{t w(B(\mathbf{y}, \sqrt{t}))} .
$$

Proof Thanks to the fact that operator $L$ is positive and self-adjoint, we have that the semigroup $\left\{K_{t}\right\}_{t \geq 0}$ is analytic on $L^{2}(d w)$, so the operator $L K_{t / 2}$ is bounded on $L^{2}(d w)$ for all $t>0$. Therefore, by the semigroup property and the definition of $L$ (here $L_{\mathbf{X}}$ denotes the action of $L$ with respect to the $\mathbf{x}$-variable) we have

$$
L_{\mathbf{x}} k_{t}(\mathbf{x}, \mathbf{y})=L_{\mathbf{x}} \int_{\mathbb{R}^{N}} k_{t / 2}(\mathbf{x}, \mathbf{z}) k_{t / 2}(\mathbf{z}, \mathbf{y}) d w(\mathbf{z})=\left(\left(L K_{t / 2}\right) k_{t / 2}(\cdot, \mathbf{y})\right)(\mathbf{x}) .
$$

Consequently, by the Cauchy-Schwarz inequality we have

$$
\begin{aligned}
\left\langle L k_{t}(\cdot, \mathbf{y}), k_{t}(\cdot, \mathbf{y})\right\rangle & =\left\langle L K_{t / 2}\left(k_{t / 2}(\cdot, \mathbf{y})\right)(\cdot), k_{t}(\cdot, \mathbf{y})\right\rangle \\
& \leq\left\|k_{t}(\cdot, \mathbf{y})\right\|_{L^{2}(d w)}\left\|L K_{t / 2}\left(k_{t / 2}(\cdot, \mathbf{y})\right)(\cdot)\right\|_{L^{2}(d w)}
\end{aligned}
$$

By Theorem 2 and (26) we obtain

$$
\left\|k_{t}(\cdot, \mathbf{y})\right\|_{L^{2}(d w)} \leq \frac{C}{w(B(\mathbf{y}, \sqrt{t}))^{1 / 2}} .
$$

Moreover, holomorphy of $\left\{K_{t}\right\}_{t \geq 0}$ together with Theorem 2 lead us to

$$
\left\|L K_{t / 2}\left(k_{t / 2}(\cdot, \mathbf{y})\right)(\cdot)\right\|_{L^{2}(d w)} \leq C \frac{1}{t}\left\|k_{t / 2}(\cdot, \mathbf{y})\right\|_{L^{2}(d w)} \leq C^{\prime} \frac{1}{t w(B(\mathbf{y}, \sqrt{t}))^{1 / 2}} .
$$

The claim is a consequence of (103) together with (104) and (105).

Now we are ready prove that the condition (D) holds for the potential $V$ satisfying the reverse Hölder inequality (27). Fix $\mathbf{y} \in \mathbb{R}^{N}$ and $0<t \leq d(Q)^{2}$. For any $r>0$, by Cauchy-Schwarz inequality, (26), and Theorem 2, we obtain

$$
\begin{aligned}
I=\left(\int_{\mathbb{R}^{N}} k_{t}(\mathbf{x}, \mathbf{y}) d w(\mathbf{x})\right)^{2} \leq & 2\left(\int_{\|\mathbf{x}-\mathbf{y}\| \leq r} k_{t}(\mathbf{x}, \mathbf{y}) d w(\mathbf{x})\right)^{2} \\
& +2\left(\int_{\|\mathbf{x}-\mathbf{y}\|>r} k_{t}(\mathbf{x}, \mathbf{y}) d w(\mathbf{x})\right)^{2} \\
\leq & C w(B(\mathbf{y}, r)) \int_{\|\mathbf{x}-\mathbf{y}\| \leq r} k_{t}(\mathbf{x}, \mathbf{y})^{2} d w(\mathbf{x})+C t r^{-2} .
\end{aligned}
$$

By (102) and the comment above (102) we have $k_{t}(\cdot, \mathbf{y}) \in \mathcal{D}(L)$. Therefore

$$
\mathbf{Q}\left(k_{t}(\cdot, \mathbf{y}), k_{t}(\cdot, \mathbf{y})\right)=\left\langle L k_{t}(\cdot, \mathbf{y}), k_{t}(\cdot, \mathbf{y})\right\rangle \text {. }
$$


Consequently, using (46), then the Fefferman-Phong inequality (53), we get

$$
\begin{aligned}
I & \leq C w(B(\mathbf{y}, r)) m(\mathbf{y})^{-2}(1+r m(\mathbf{y}))^{\frac{2 \kappa}{1+\kappa}} \int_{\mathbb{R}^{N}} k_{t}(\mathbf{x}, \mathbf{y})^{2} m(\mathbf{x})^{2} d w(\mathbf{x})+C t r^{-2} \\
& \leq C w(B(\mathbf{y}, r)) m(\mathbf{y})^{-2}(1+r m(\mathbf{y}))^{\frac{2 \kappa}{1+\kappa}}\left\langle L k_{t}(\cdot, \mathbf{y}), k_{t}(\cdot, \mathbf{y})\right\rangle+C t r^{-2} .
\end{aligned}
$$

By (101) and (10) we get

$$
\begin{aligned}
I & \leq C \frac{w(B(\mathbf{y}, r))}{t w(B(\mathbf{y}, \sqrt{t}))} m(\mathbf{y})^{-2}(1+r m(\mathbf{y}))^{\frac{2 \kappa}{1+\kappa}}+C t r^{-2} \\
& \leq C t^{-1}\left(r^{\mathbf{N}} t^{-\mathbf{N} / 2}+r^{N} t^{-N / 2}\right) m(\mathbf{y})^{-2}(1+r m(\mathbf{y}))^{\frac{2 \kappa}{1+\kappa}}+C t r^{-2} .
\end{aligned}
$$

If we plug in $r=t^{\frac{1+\varepsilon}{2}} m(\mathbf{y})^{\varepsilon}$, we get

$$
\begin{gathered}
I \leq C\left(t^{\mathbf{N} \varepsilon / 2-1} m(\mathbf{y})^{\mathbf{N} \varepsilon-2}+t^{N \varepsilon / 2-1} m(\mathbf{y})^{N \varepsilon-2}\right) \\
\left(1+t^{1 / 2+\varepsilon / 2} m(\mathbf{y})^{1+\varepsilon}\right)^{2 \kappa /(1+\kappa)}+C t^{-\varepsilon} m(\mathbf{y})^{-2 \varepsilon} .
\end{gathered}
$$

Recall that $m(\mathbf{y}) \sim d(Q)^{-1}$ for $\mathbf{y} \in Q^{* * * *}$. Hence for $t>d(Q)^{2} \sim m(\mathbf{y})^{-2}$ if we take $\varepsilon$ small enough, we get

$$
I \leq C t^{-\varepsilon_{1}} m(\mathbf{y})^{-2 \varepsilon_{1}} \text { for some } \varepsilon_{1}>0
$$

which ends the proof.

\subsection{Verification of Condition (K)}

Assume that $V \in \mathrm{RH}^{q}(d w), q>\max \left(1, \frac{\mathbf{N}}{2}\right)$. Thanks to Hölder's inequality we have

$$
\begin{aligned}
I= & \int_{0}^{2 t} \int_{Q^{* * *}} V(\mathbf{y}) \mathcal{G}_{2 s / c}(\mathbf{x}, \mathbf{y}) d w(\mathbf{y}) d s \\
\leq & \int_{0}^{2 t}\left(\frac{1}{w\left(Q^{* * *}\right)} \int_{Q^{* * *}} V(\mathbf{y})^{q} d w(\mathbf{y})\right)^{1 / q} w\left(Q^{* * *}\right)^{1 / q} \\
& \left(\int_{Q^{* * *}} \mathcal{G}_{2 s / c}(\mathbf{x}, \mathbf{y})^{q^{\prime}} d w(\mathbf{y})\right)^{1 / q^{\prime}} d s .
\end{aligned}
$$

Note that for $\mathbf{y} \in Q^{* * *}$ we have $w(B(\mathbf{y}, d(Q))) \sim w\left(Q^{* * *}\right)$, therefore, by Theorem 2, we have

$$
\begin{aligned}
& w\left(Q^{* * *}\right)^{1 / q}\left(\int_{Q^{* * *}} \mathcal{G}_{2 s / c}(\mathbf{x}, \mathbf{y})^{q^{\prime}} d w(\mathbf{y})\right)^{1 / q^{\prime}} \\
& \quad \leq C\left(\int_{Q^{* * *}} \frac{w(B(\mathbf{y}, d(Q)))^{q^{\prime} / q}}{w(B(\mathbf{y}, \sqrt{s}))^{q^{\prime}-1}} \frac{1}{w(B(\mathbf{x}, \sqrt{s}))} e^{-c q^{\prime} d(\mathbf{x}, \mathbf{y})^{2} /(2 s)} d w(\mathbf{y})\right)^{1 / q^{\prime}}
\end{aligned}
$$


Recall that $\sqrt{s} \leq \sqrt{2 t} \leq \sqrt{2} d(Q)$ in the assumption (K), so, thanks to (10), we have

$$
\frac{w(B(\mathbf{y}, d(Q)))^{q^{\prime} / q}}{w(B(\mathbf{y}, \sqrt{s}))^{q^{\prime}-1}}=\frac{w(B(\mathbf{y}, d(Q)))^{q^{\prime} / q}}{w(B(\mathbf{y}, \sqrt{s}))^{q^{\prime} / q}} \leq C\left(\frac{d(Q)}{\sqrt{s}}\right)^{\left(\mathbf{N} q^{\prime}\right) / q} .
$$

Consequently, (108) and (109) lead us to

$$
I \leq C \int_{0}^{2 t}\left(\frac{d(Q)}{\sqrt{s}}\right)^{\mathbf{N} / q}\left(\frac{1}{w\left(Q^{* * *}\right)} \int_{Q^{* * *}} V(\mathbf{y})^{q} d w(\mathbf{y})\right)^{1 / q} d s .
$$

Applying the reverse Hölder inequality (27) and the fact that $q>\max \left(1, \frac{\mathbf{N}}{2}\right)$ we have

$$
I \leq C t\left(\frac{d(Q)}{\sqrt{t}}\right)^{\mathbf{N} / q} \frac{1}{w\left(Q^{* * *}\right)} \int_{Q^{* * *}} V(\mathbf{y}) d w(\mathbf{y}) \leq C\left(\frac{d(Q)}{\sqrt{t}}\right)^{-\gamma}
$$

where, in the last step, the fact that the measures $\mu$ and $w$ are doubling and the definition of $Q \in \mathcal{Q}$ by the stopping-time condition (48) were utilize. The proof is finished (we set $\delta=\gamma / 2$ ).

Acknowledgements The author would like to thank Jacek Dziubański for his helpful comments and suggestions. Research supported by the National Science Centre, Poland (Narodowe Centrum Nauki), Grant2017/25/B/ST1/00599. The author would like thank the anonymous reviewers for their careful reading of our manuscript and their comments and suggestions.

\section{Compliance with Ethical Standards}

Conflicts of interest The authors declare that they have no conflict of interest.

Open Access This article is licensed under a Creative Commons Attribution 4.0 International License, which permits use, sharing, adaptation, distribution and reproduction in any medium or format, as long as you give appropriate credit to the original author(s) and the source, provide a link to the Creative Commons licence, and indicate if changes were made. The images or other third party material in this article are included in the article's Creative Commons licence, unless indicated otherwise in a credit line to the material. If material is not included in the article's Creative Commons licence and your intended use is not permitted by statutory regulation or exceeds the permitted use, you will need to obtain permission directly from the copyright holder. To view a copy of this licence, visit http://creativecommons.org/licenses/by/4.0/.

\section{References}

1. Amri, B.: Riesz transforms for Dunkl Hermite expansions. J. Math. Anal. Appl. 423(1), 646-659 (2015)

2. Amri, B., Hammi, A.: Dunkl-Schrödinger operators. Complex Anal. Oper. Theory (2018)

3. Amri, B., Hammi, A.: Semigroup and Riesz transform for the Dunkl-Schrödinger operators. Semigroup Forum 101(3), 507-533 (2020)

4. Anker, J-Ph, Salem, N.B., Dziubański, J., Hamda, N.: The Hardy space $H^{1}$ in the rational Dunkl setting. Constr. Approx. 42(1), 93-128 (2015)

5. Anker, J-Ph, Dziubański, J., Hejna, A.: Harmonic functions, conjugate harmonic functions and the Hardy space $H^{1}$ in the rational Dunkl setting. J. Fourier Anal. Appl. 25, 2356-2418 (2019) 
6. Coifman, R.R.: A real variable characterization of $H^{p}$. Stud. Math. 51, 269-274 (1974)

7. de Jeu, M.F.E.: The Dunkl transform. Invent. Math. 113, 147-162 (1993)

8. de Jeu, M., Rösler, M.: Asymptotic analysis for the Dunkl kernel. J. Approx. Theory 119(1), 110-126 (2002)

9. Dunkl, C.F.: Reflection groups and orthogonal polynomials on the sphere. Math. Z. 197(1), 33-60 (1988)

10. Dunkl, C.F.: Differential-difference operators associated to reflection groups. Trans. Am. Math. 311(1), 167-183 (1989)

11. Dunkl, C.F.: Hankel transforms associated to finite reflection groups. In: Proc. of the Special Session on Hypergeometric Functions on Domains of Positivity, Jack Polynomials and Applications. Proceedings, Tampa 1991, Contemp. Math., vol. 138, pp. 123-138 (1989)

12. Dunkl, C.F.: Integral kernels with reflection group invariance. Can. J. Math. 43(6), 1213-1227 (1991)

13. Dziubański, J., Hejna, A.: Remark on atomic decompositions for Hardy space $H^{1}$ in the rational Dunk1 setting. Studia Math. 251(1), 89-110 (2020)

14. Dziubański, J., Zienkiewicz, J.: Hardy spaces associated with some Schrödinger operators. Studia Math. 126(2), 149-160 (1997)

15. Dziubański, J., Zienkiewicz, J.: Hardy space $H^{1}$ associated to Schrödinger operator with potential satisfying reverse Hölder inequality. Rev. Mat. Iberoamericana 15(2), 279-296 (1999)

16. Dziubański, J., Zienkiewicz, J.: $H^{p}$ spaces for Schrödinger operators. In: Fourier Analysis and Related Topics (Bedlewo, 2000), vol. 56, pp. 45-53. Banach Center Publ., Polish Acad. Sci. Inst. Math., Warsaw (2002)

17. Dziubański, J., Zienkiewicz, J.: $H^{p}$ spaces associated with Schrödinger operators with potentials from reverse Hölder classes. Colloq. Math. 98(1), 5-38 (2003)

18. Dziubański, J., Zienkiewicz, J.: Hardy spaces $H^{1}$ for Schrödinger operators with certain potentials. Studia Math. 164, 39-53 (2004)

19. Fefferman, C., Stein, E.M.: $H^{p}$ spaces of several variables. Acta Math. 129(3-4), 137-193 (1972)

20. Fefferman, C.: The uncertainty principle. Bull. Am. Math. Soc. 9(2), 129-206 (1983)

21. Gallardo, L., Rejeb, C.: A new mean value property for harmonic functions relative to the DunklLaplacian operator and applications. Trans. Am. Math. Soc. 368(5), 3727-3753 (2015)

22. Grafakos, L.: Classical Fourier Analysis, 3rd edition, Graduate Texts in Mathematics, 249. Springer, New York (2014)

23. Goldberg, D.: A local version of real Hardy spaces. Duke Math. J. 46(1), 27-42 (1979)

24. Hejna, A.: Hardy spaces for the Dunkl harmonic oscillator. Math. Nachr. 293(11), 2112-2139 (2020)

25. Hofmann, S., Lu, G., Mitrea, D., Mitrea, M., Yan, L.: Hardy spaces associated to non-negative selfadjoint operators satisfying Davies-Gaffney estimates. Mem. Am. Math. Soc. 21(1007), vi+78 (2011)

26. Latter, R.: A characterization of $H^{p}\left(R^{n}\right)$ in terms of atoms. Studia Math. 62(1), 93-101 (1978)

27. Maslouhi, M., Bentaleb, A., Elhassani, M.E.: A sequence of remarkable Poincaré inequalities for Dunkl operators. Integr Transforms Spec. Funct. 30(2), 157-165 (2019)

28. Nowak, A., Stempak, K.: Riesz transforms for the Dunkl harmonic oscillator. Math. Z. 262(3), 539-556 (2009)

29. Rösler, M.: Generalized Hermite polynomials and the heat equation for Dunkl operators. Commun. Math. Phys. 192, 519-542 (1998)

30. Rösler, M.: Positivity of Dunkl's intertwining operator. Duke Math. J. 98(3), 445-463 (1999)

31. Rösler, M.: A positive radial product formula for the Dunkl kernel. Trans. Am. Math. Soc. 355(6), 2413-2438 (2003)

32. Rösler, M.:Dunkl operators (theory and applications). In: Koelink, E., Van Assche, W. (eds.) Orthogonal Polynomials and Special Functions (Leuven, 2002), Lect. Notes Math. vol. 1817, pp. 93-135. Springer (2003)

33. Rösler, M., Voit, M.: Dunkl theory, convolution algebras, and related Markov processes. In: Graczyk, P., Rösler, M., Yor M. (eds.) Harmonic and Stochastic Analysis of Dunkl Processes. Travaux en cours, vol. 71, pp. 1-112. Hermann, Paris (2008)

34. Stein, E.M.: Harmonic Analysis (Real Variable Methods, Orthogonality and Oscillatory Integrals), Princeton Math. Series, vol. 43. Princeton Univ. Press (1993)

35. Shen, Z.: On the Neumann problem for Schrödinger operators in Lipschitz domains. Indiana Univ. Math. J. 43(1), 143-176 (1994)

36. Shen, Z: $L^{p}$ estimates for Schrödinger operators with certain potentials. Ann. Inst. Fourier (Grenoble) 45(2), 513-546 (1995) 
37. Stein, E.M., Weiss, G.: On the theory of harmonic functions of several variables. I. The theory of $H^{p}$-spaces. Acta Math. 103, 25-62 (1960)

38. Thangavelu, S., Xu, Y.: Convolution operator and maximal function for the Dunkl transform. J. Anal. Math. 97, 25-55 (2005)

39. Triméche, K.: Paley-Wiener theorems for the Dunkl transform and Dunkl translation operators. Integr. Transforms Spec. Funct. 13(1), 17-38 (2002)

40. Velicu, A.: Sobolev-type inequalities for Dunkl operators. J. Funct. Anal. 279(7), 108695 (2020)

41. Velicu, A.: Logarithmic Sobolev inequalities for Dunkl operators, arXiv:1910.06857

Publisher's Note Springer Nature remains neutral with regard to jurisdictional claims in published maps and institutional affiliations. 